\title{
IDENTIFICATION OF A SOURCE TERM IN A SEMILINEAR EVOLUTION DELAY EQUATION
}

\author{
BY
}

\author{
ALFREDO LORENZI* ${ }^{*}$ and IOAN I. VRABIE
}

\begin{abstract}
An existence, uniqueness and continuous dependence on the data result for a source term identification problem in a semilinear functional delay differential equation in a general Banach space is established. As additional condition, it is assumed that the mean of the solution, with respect to a non-atomic Borel measure, is a preassigned element in the domain of the linear part of the right-hand side of the equation. Two applications to source identification, one in a parabolic functional delay equation and another one in a hyperbolic delay equation, are also discussed.

Mathematics Subject Classification 2010: 34K29, 34K30, 35K58, 35L02, 47D06, $47 \mathrm{H} 10$.

Key words: identification problem, first-order semilinear functional differential delay equation, unknown source, $C_{0}$-semigroup of contractions, parabolic functional delay equation, first-order hyperbolic functional delay equation.
\end{abstract}

\section{Introduction}

Let $X$ be a Banach space whose norm is denoted by $\|\cdot\|$, let $A: D(A) \subseteq$ $X \rightarrow X$ be the infinitesimal generator of a $C_{0}$-semigroup of contractions $\{S(t) ; t \geq 0\}$, let $\tau \in(0,1)$, let $\varphi:[0,1] \times C([-\tau, 0] ; X) \rightarrow \mathbb{R}$ be a nonlinear $C^{1}$-functional, let $f:[0,1] \times C([-\tau, 0] ; X) \rightarrow X$ be a $C^{1}$-function and let $\psi \in\left[W^{1,1}([0,1] ; \mathbb{R}) \backslash\{0\}\right]$ be a positive function. Throughout, if

\footnotetext{
${ }^{*}$ The first author is a member of G.N.A.M.P.A. of the Italian Istituto Nazionale di Alta Matematica.

Note. Regretfully, on 9 November 2013, the first author, Professor Alfredo Lorenzi, passed away suddenly.
} 
$u \in C([-\tau, 1] ; X)$ and $t \in[0,1], u_{t}$ denotes the function in $C([-\tau, 0] ; X)$, defined by

$$
u_{t}(s):=u(t+s)
$$

for each $s \in[-\tau, 0]$.

In this paper we prove an existence, uniqueness and continuous dependence on the data result for a source term identification problem:

$(\mathcal{J P})$ given $h \in C^{1}([-\tau, 0] ; X)$ and $\xi \in X$, find $z \in X$ and a mild solution $u:[0,1] \rightarrow X$ of the Cauchy Problem

$$
\begin{cases}u^{\prime}(t)=A u(t)+\varphi\left(t, u_{t}\right) z+f\left(t, u_{t}\right), & t \in[0,1], \\ u(t)=h(t), & t \in[-\tau, 0],\end{cases}
$$

satisfying the additional condition

$$
\int_{0}^{1} \psi(t) u(t) d t=\xi
$$

The constant delayed case, i.e.

$$
\begin{cases}u^{\prime}(t)=A u(t)+\varphi(t, u(t), u(t-\tau)) z+f(t, u(t), u(t-\tau)), & t \in[0,1] \\ u(t)=h(t), & t \in[-\tau, 0]\end{cases}
$$

where $\varphi:[0,1] \times X \times X \rightarrow \mathbb{R}$ and $f:[0,1] \times X \times X \rightarrow X$, was considered in LorEnZI-VRABIE [9]. Since

$$
\left\{\begin{array}{l}
\varphi(t, u(t), u(t-\tau))=\varphi\left(t, \delta(s-0) u_{t}, \delta(s-\tau) u_{t}\right)=\widetilde{\varphi}\left(t, u_{t}\right), \\
f(t, u(t), u(t-\tau))=f\left(t, \delta(s-0) u_{t}, \delta(s-\tau) u_{t}\right)=\widetilde{f}\left(t, u_{t}\right)
\end{array}\right.
$$

$\delta(s+a)$ being the Dirac measure concentrated at $a,(1.3)$ is a particular case of (1.1).

Previous results on the non-delayed linear versions of (1.3) was analyzed by LORENZI and VRABIE [6], while the semilinear non-delayed case with $\varphi$ depending only on $u, \psi(t)=1$ for $t \in[0,1]$, and $f$ independent of $u_{t}$, was considered in LORENZI and VRABIE [7]. The semilinear non-delayed case with $\varphi$ depending on $t$ and $\psi$ being arbitrary in $W^{1,1}$ was analyzed in LORENZI and VRABIE [8]. Identification problems, aimed at recovering a time-depending function in delay functional differential equations were studied by Di BLASIO and Lorenzi [3] and [5], in the case of an unknown factor in the source, and in [4], in the case of an unknown convolution kernel. 
It should be emphasized that, unlike in LORENZI and VRABIE [7], where $A$ is assumed to generate a compact $C_{0}$-semigroup which is an abstract parabolicity condition, here - following the same strategy as in LORENZI and VRABIE [9] - we merely assume that $(I-A)^{-1}$ is compact, condition which is satisfied in both parabolic and hyperbolic problems. We recall that, if the $C_{0}$-semigroup generated by $A$ is compact, then $(I-A)^{-1}$ is compact, but not conversely. See for instance PAZY [11, Theorem 6.2.1] or VRABIE [14, p. 134].

Definition 1.1. By definition, a strict solution of the equation (1.1) is a function $u \in C([-\tau, 1] ; X) \cap C^{1}([0,1] ; X) \cap C([0,1] ; D(A))^{1}$, satisfying

$$
u^{\prime}(t)=A u(t)+\varphi\left(t, u_{t}\right) z+f\left(t, u_{t}\right)
$$

for each $t \in[0,1]$ as well as $u(t)=h(t)$ for $t \in[-\tau, 0]$.

Accordingly, by a strict solution of $(\mathcal{J P})$ we mean a pair $(z, u)$ such that $u$ is a strict solution of the Cauchy problem (1.1) and, in addition, $u$ verifies (1.2).

The paper is divided into seven sections. Section 2 is devoted to some preliminaries while, in Section 3, we state our main existence result concerning mild solutions, i.e. Theorem 3.1. Section 4 is concerned with the proof of Theorem 3.1, Section 5 deals with a uniqueness and continuous dependence result, i.e., Theorem 6.1. In Sections 6 and 7 we include two examples interesting by themselves. The first one refers to an identification problem for a parabolic semilinear delay equation, while the latter is devoted to an identification problem for a hyperbolic delay problem.

\section{Preliminaries}

\subsection{The compactness argument}

Let $\xi \in X$ be fixed, let $\mathcal{F} \subseteq L^{1}(0,1 ; X), g \in \mathcal{F}$ and let us consider the evolution equation

$$
\left\{\begin{array}{l}
u^{\prime}(t)=A u(t)+g(t), \quad t \in[0,1] \\
u(0)=\xi
\end{array}\right.
$$

\footnotetext{
${ }^{1}$ Hereafter $C([0,1] ; D(A))$ is the space of all continuous functions from $[0,1]$ to $D(A)$, the latter being endowed with the usual graph norm $\|\cdot\|_{A}$, defined by $\|u\|_{A}=\|u\|+\|A u\|$ for each $u \in D(A)$.
} 
Throughout $\xi \in X$ is assumed to be fixed and we denote by $u_{g}$ the unique mild solution of (2.1) corresponding to $g \in \mathcal{F}$, i.e.,

$$
u_{g}(t)=S(t) \xi+\int_{0}^{t} S(t-s) g(s) d s
$$

for $t \in[0,1]$, and by

$$
\mathcal{M}(\mathcal{F})=\left\{u_{g} ; g \in \mathcal{F}\right\},
$$

the set of mild solutions of (2.1) corresponding to all $g \in \mathcal{F}$.

A set $\mathcal{F} \subseteq L^{1}(0,1 ; X)$ is called Lebesgue-uniformly integrable if for each $\varepsilon>0$ there exists $\delta(\varepsilon)>0$ such that, for each measurable subset $E \subseteq[0,1]$ with Lebesgue measure $\lambda(E) \leq \delta(\varepsilon)$, we have

$$
\int_{E}\|g(t)\| d t \leq \varepsilon
$$

uniformly for $g \in \mathcal{F}$.

The next compactness result, for $A$ being a nonlinear and $m$-dissipative operator and $\mathcal{F}$ being bounded in $C([0,1] ; X)$, was proved by VRABIE [12]. The extension to the more general case when $\mathcal{F}$ is uniformly integrable in $L^{1}(0,1 ; X)$ was obtained by Mitidieri and VRABIE [10]. We confine ourselves to state below only the linear variant which is exactly what we need for our later purposes. For details see VRABIE [14, Theorem 8.5.1, p. 197].

Theorem 2.1. Let $A: D(A) \subseteq X \rightarrow X$ be the infinitesimal generator of a $C_{0}$-semigroup $\{S(t) ; t \geq 0\}$ and let us assume that $(I-A)^{-1}$ is compact. Let $\mathcal{F} \subseteq L^{1}(0,1 ; X)$ be a Lebesgue-uniformly integrable set and let $\xi \in X$ be fixed. Then, the following conditions are equivalent:

$\left(c_{1}\right)$ the set $\mathcal{M}(\mathcal{F})$ is equicontinuous from the right on $[0,1)$;

$\left(c_{2}\right)$ the set $\mathcal{M}(\mathcal{F})$ is relatively compact in $C([0,1] ; X)$.

\subsection{An equivalent problem}

We reformulate the identification problem $(\mathcal{J P})$ as an equivalent nonlinear delay evolution equation more convenient for our later purposes. Let us assume for the time being that $(z, u)$ is a strict solution of $(\mathcal{J P})$. Clearly

$$
\int_{0}^{1} \psi(t) u^{\prime}(t) d t=\psi(1) u(1)-\psi(0) h(0)-\int_{0}^{1} \psi^{\prime}(t) u(t) d t .
$$


Since $A$ is closed, from VRABIE [14, Hille's Theorem 1.2.2, p. 8], we have

$$
\int_{0}^{1} \psi(t) A u(t) d t=A \int_{0}^{1} \psi(t) u(t) d t=A \xi .
$$

Multiplying both sides of the equation in (1.1) by $\psi(t)$, integrating over $[0,1]$, taking into account the equalities above, we deduce

$$
\begin{aligned}
& \psi(1) u(1)-\psi(0) h(0)-\int_{0}^{1} \psi^{\prime}(t) u(t) d t \\
& =\left(\int_{0}^{1} \psi(t) \varphi\left(t, u_{t}\right) d t\right) z+A \xi+\int_{0}^{1} \psi(t) f\left(t, u_{t}\right) d t
\end{aligned}
$$

Let us assume further that, for each $v \in C([-\tau, 0] ; X)$, we have

$$
\left|\int_{0}^{1} \psi(t) \varphi(t, v) d t\right| \geq m>0
$$

where $m$ is independent of $v$. Clearly, in this case, we get

$$
\left|\int_{0}^{1} \psi(t) \varphi\left(t, u_{t}\right) d t\right| \geq m>0
$$

for each $u \in C([-\tau, 1] ; X),(2.2)$ and $(2.3)$ being equivalent.

Under the circumstances mentioned above, we observe that

$$
z=F(u)
$$

where $F: C([-\tau, 1] ; X) \rightarrow X$ (here $h$ and $\xi$ are fixed) is defined by

$$
F(u)=J(u)\left[\eta+\psi(1) u(1)-\int_{0}^{1} \psi^{\prime}(t) u(t) d t-\int_{0}^{1} \psi(t) f\left(t, u_{t}\right) d t\right],
$$

$\eta \in X$ and $J: C([-\tau, 1] ; X) \rightarrow \mathbb{R}$ being given by

$$
\left\{\begin{array}{l}
\eta=-A \xi-\psi(0) h(0) \\
J(u):=\left(\int_{0}^{1} \psi(t) \varphi\left(t, u_{t}\right) d t\right)^{-1}
\end{array}\right.
$$

Whence, if $(z, u)$ is a strict solution of $(\mathcal{J P})$, then $u$ is a strict solution of the problem

$$
\begin{cases}u^{\prime}(t)=A u(t)+\varphi\left(t, u_{t}\right) F(u)+f\left(t, u_{t}\right), & t \in[0,1] \\ u(t)=h(t), & t \in[-\tau, 0],\end{cases}
$$


$F(u)$ being defined by $(2.5)$.

Under some additional easy to verify conditions, the converse statement holds also true. Namely, we have:

Lemma 2.1. Let (2.2) be satisfied, let $A$ be injective and let $u$ be a strict solution of the problem (2.7), where $F(u)$ is defined by (2.5), while $\eta$ and $J(u)$ are defined by (2.6). Then $u$ satisfies the additional condition (1.2), and so $(F(u), u)$ is a strict solution of $(\mathrm{JP})$.

Since the proof of Lemma 2.1 follows the very same arguments as in the proof of Lemma 2.1 in LoRENZI and VRABIE [9], we do not enter into details.

Following LORENZI and VRABIE [9], we introduce two concepts of solution for our identification problem. We start with:

Definition 2.1. By a generalized solution of the identification problem $(\mathcal{J P})$ we mean a function $u \in C([-\tau, 1] ; X)$ satisfying

$$
u(t)=h(t)
$$

for $t \in[-\tau, 0]$ and

$$
u(t)=h(0)+\int_{0}^{t} S(t-s) \varphi\left(s, u_{s}\right) F(u) d s+\int_{0}^{t} S(t-s) f\left(s, u_{s}\right) d s
$$

for $t \in[0,1]$, where $F(u)$ is given by $(2.5)$.

Definition 2.2. By a mild solution of the identification problem (JP) we mean a pair $(z, u) \in X \times C([-\tau, 1] ; X)$ satisfying

$$
u(t)=h(t)
$$

for $t \in[-\tau, 0]$,

$$
u(t)=h(0)+\int_{0}^{t} S(t-s)\left[\varphi\left(s, u_{s}\right) z+f\left(s, u_{s}\right)\right] d s
$$

for $t \in[0,1]$, and the additional condition (1.2).

Remark 2.1. Clearly, each strict solution of $(\mathcal{J P})$ is a mild solution too, but the converse statement is no longer true. Moreover, each mild solution of $(\mathcal{J P})$ is a generalized solution too. The converse of the last statement holds also true under the additional condition that $A^{-1} \in \mathcal{L}(X)$. We notice that this condition is satisfied, whenever $A$ generates a $C_{0}$-semigroup having exponential decay. 
Indeed, we have:

Lemma 2.2. Let us assume that $A^{-1} \in \mathcal{L}(X), \xi \in D(A), h \in C([-\tau, 0] ; X)$ satisfies $h(0) \in D(A)$ and (2.4) holds true. If $u$ is a generalized solution of the identification problem (JP), then $u$ is a mild solution of the problem (1.1), with $z=F(u)$ given by (2.5), and, in addition, $u$ satisfies (1.2).

Also the proof of Lemma 2.2 is very similar to the proof of the corresponding Lemma 2.2 in LORENZI and VRABIE [9] and therefore we omit it.

\subsection{The main assumptions}

The hypotheses we need are listed below.

$\left(H_{A}\right) \quad A: D(A) \subseteq X \rightarrow X$ generates a $C_{0}$-semigroup $\{S(t) ; t \geq 0\}$ and

$\left(A_{1}\right)$ the resolvent of $A$, i.e. $(I-A)^{-1}$ is compact;

$\left(A_{2}\right)$ there exists $\omega>0$ such that $\|S(t)\| \leq e^{-\omega t}$ for each $t \geq 0$;

$\left(H_{\varphi}\right) \varphi:[0,1] \times C([-\tau, 0] ; X) \rightarrow \mathbb{R}$ is a functional satisfying

$\left(\varphi_{1}\right)$ there exists $\ell>0$ such that

$$
\begin{aligned}
& \mid \varphi(t, v)-\varphi(\widetilde{t}, \widetilde{v}) \| \leq \ell\left[|t-\widetilde{t}|+\|v-\widetilde{v}\|_{C([-\tau, 0] ; X)}\right] \\
& (t, v),(\widetilde{t}, \widetilde{v}) \in[0,1] \times C([-\tau, 0] ; X)
\end{aligned}
$$

$\left(\varphi_{2}\right) \varphi$ is bounded from below by a strictly positive number and it is bounded from above, i.e.,

$$
\begin{aligned}
& m_{1}:=\inf \{\varphi(t, v) ;(t, v) \in[0,1] \times C([-\tau, 0] ; X)\}>0 \\
& m_{2}:=\sup \{\varphi(t, v) ;(t, v) \in[0,1] \times C([-\tau, 0] ; X)\}<+\infty ;
\end{aligned}
$$

$\left(H_{f}\right) f:[0,1] \times C([-\tau, 0] ; X) \rightarrow X$ is a $C^{1}$-function and there exist two constants $\ell_{i}>0, i=1,2$, such that

$\left(f_{1}\right)\|f(t, v)-f(\widetilde{t}, \widetilde{v})\| \leq \ell_{1}\left[|t-\widetilde{t}|+\|v-\widetilde{v}\|_{C([-\tau, 0] ; X)}\right]$ for each $(t, v)$, $(\widetilde{t}, \widetilde{v}) \in[0,1] \times C([-\tau, 0] ; X)$

$\left(f_{2}\right)\|f(t, 0)\| \leq \ell_{2}$ for each $t \in[0,1]$; 
$\left(H_{\psi}\right) \psi \in\left[W^{1,1}([0,1] ; \mathbb{R}) \backslash\{0\}\right], \psi(t) \geq 0$ for each $t \in[0,1]$.

Remark 2.2. ( $i$ ) If $A$ satisfies $\left(A_{2}\right)$ then $A+\omega I$ is dissipative - see PAZY [11, Theorem 4.3] - and thus $A^{-1} \in \mathcal{L}(X)$. As a consequence, $A$ is injective.

(ii) If $\left(\varphi_{2}\right)$ in $\left(H_{\varphi}\right)$ and $\left(H_{\psi}\right)$ hold, then there exists a constant $m>0$ such that

$$
\int_{0}^{1} \psi(t) \varphi\left(t, u_{t}\right) d t \geq m
$$

for each $u \in C([-\tau, 1] ; X)$. Indeed, if $u \in C([-\tau, 1] ; X)$, then

$$
\int_{0}^{1} \psi(t) \varphi\left(t, u_{t}\right) d t \geq m_{1} \int_{0}^{1} \psi(t) d t:=m>0 .
$$

So $J$, defined by (2.6), satisfies

$$
|J(u)| \leq \frac{1}{m}
$$

for each $u \in C([-\tau, 1] ; X)$.

\subsection{Preliminary estimates}

Proposition 2.1. Let $\left(H_{\varphi}\right),\left(H_{f}\right)$ and $\left(H_{\psi}\right)$ be satisfied. Then the operator $F$, defined by (2.5), with $J$ being given by (2.6), is Lipschitz continuous on bounded subsets in

$$
C_{[h]}([-\tau, 1] ; X)=\{u \in C([-\tau, 1] ; X) ; u(t)=h(t) \text { for } t \in[-\tau, 0]\}
$$

and there exist two constants $L_{0}>0$ and $M_{0}>0$ such that

$$
\|F(u)\| \leq L_{0}\|u\|_{C([-\tau, 1] ; X)}+M_{0}
$$

for each $u \in C_{[h]}([-\tau, 1] ; X)$.

Proof. Let us observe first that $F$ is decomposed as

$$
F(u)=J(u) P(u)
$$

for each $u \in C_{[h]}([-\tau, 0] ; X)$, where $J: C([-\tau, 1] ; X) \rightarrow \mathbb{R}$ is defined by (2.6) and $P: C_{[h]}([-\tau, 1] ; X \rightarrow X$ is given by

$$
P(u):=\eta+\psi(1) u(1)-\int_{0}^{1} \psi^{\prime}(t) u(t) d t-\int_{0}^{1} \psi(t) f\left(t, u_{t}\right) d t
$$


for each $u \in C_{[h]}([-\tau, 0] ; X), \eta$ being defined in (2.6), i.e. $\eta=-A \xi-$ $\psi(0) h(0)$.

We begin by showing that the mapping $u \mapsto J(u)$, defined by by (2.6), is Lipschitz with constant $\frac{\ell\|\psi\|_{L^{1}(0,1 ; \mathbb{R})}}{m^{2}}$. Indeed, from $\left(H_{\varphi}\right)$ and $\left(H_{\psi}\right)$ and (ii) in Remark 2.2, we deduce

$$
\begin{aligned}
& |J(u)-J(\widetilde{u})| \leq\left|\frac{1}{\int_{0}^{1} \psi(t) \varphi\left(t, u_{t}\right) d t}-\frac{1}{\int_{0}^{1} \psi(t) \varphi\left(t, \widetilde{u}_{t}\right) d t}\right| \\
& =\left|\frac{\int_{0}^{1} \psi(t) \varphi\left(t, \widetilde{u}_{t}\right) d t-\int_{0}^{1} \psi(t) \varphi\left(t, u_{t}\right) d t}{\int_{0}^{1} \psi(t) \varphi\left(t, u_{t}\right) d t \int_{0}^{1} \psi(t) \varphi\left(t, \widetilde{u}_{t}\right) d t}\right| \leq \frac{\ell\|\psi\|_{L^{1}(0,1 ; \mathbb{R})}}{m^{2}}\|u-\widetilde{u}\|_{C([-\tau, 1] ; X)}
\end{aligned}
$$

for each $u, \widetilde{u} \in C_{[h]}([-\tau, 0] ; X)$. Next, since for each $u, \widetilde{u} \in C_{[h]}([-\tau, 0] ; X)$ and $t \in[-\tau, 0], u(t)=\widetilde{u}(t)=h(t)$, we have

$$
\|u-\widetilde{u}\|_{C([-\tau, 1] ; X)}=\|u-\widetilde{u}\|_{C([0,1] ; X) .}
$$

Therefore

$$
|J(u)-J(\widetilde{u})| \leq \frac{\ell\|\psi\|_{L^{1}(0,1 ; \mathbb{R})}}{m^{2}}\|u-\widetilde{u}\|_{C([0,1] ; X)}
$$

for each $u, \widetilde{u} \in C_{[h]}([-\tau, 0] ; X)$.

At this point, let us observe that $P$ can be rewritten as

$$
P(u)=\eta+\psi(1) u(1)-\int_{0}^{1} \psi^{\prime}(t) u(t) d t-\int_{0}^{1} \psi(t)\left[f(t, 0)+f\left(t, u_{t}\right)-f(t, 0)\right] d t,
$$

where $\eta$ is given by (2.6). So, we deduce

$$
\begin{aligned}
& \|P(u)\| \leq\|\eta\|+\left[\psi(1)+\left\|\psi^{\prime}\right\|_{L^{1}(0,1 ; \mathbb{R})}\right]\|u\|_{C([0,1] ; X)} \\
& +\|\psi\|_{L^{1}(0,1 ; \mathbb{R})}\|f(\cdot, 0)\|_{C([0,1] ; X)}+\ell_{1} \int_{0}^{1} \psi(t)\left\|u_{t}\right\|_{C([-\tau, 0] ; X)} d t \\
& \leq\left[\psi(1)+\left\|\psi^{\prime}\right\|_{L^{1}(0,1 ; \mathbb{R})}+\ell_{1}\|\psi\|_{L^{1}(0,1 ; \mathbb{R})}\right]\|u\|_{C([-\tau, 1] ; X)} \\
& +\|\eta\|+\ell_{2}\|\psi\|_{L^{1}(0,1 ; \mathbb{R})}
\end{aligned}
$$


for each $u \in C_{[h]}([-\tau, 1] ; X)$. We also have

$$
\begin{aligned}
& \|P(u)-P(\widetilde{u})\| \leq \psi(1)\|u(1)-\widetilde{u}(1)\|+\int_{0}^{1}\left|\psi^{\prime}(t)\right|\|u(t)-\widetilde{u}(t)\| d t \\
& +\int_{0}^{1} \psi(t)\left\|f\left(t, u_{t}\right)-f\left(t, \widetilde{u}_{t}\right)\right\| d t \leq\left[\psi(1)+\left\|\psi^{\prime}\right\|_{\left.L^{1}(0,1 ; \mathbb{R})\right]\|u-\widetilde{u}\|_{C([0,1] ; X)}}\right. \\
& +\ell_{1} \int_{0}^{1} \psi(t)\left[\left\|u_{t}-\widetilde{u}_{t}\right\|_{C([-\tau, 0] ; X)} d t\right. \\
& \leq\left[\psi(1)+\left\|\psi^{\prime}\right\|_{L^{1}(0,1 ; \mathbb{R})}+\ell_{1}\|\psi\|_{L^{1}(0,1 ; \mathbb{R})}\right]\|u-\widetilde{u}\|_{C([-\tau, 1] ; X)}
\end{aligned}
$$

for each $u, \widetilde{u} \in C_{[h]}([-\tau, 1] ; X)$ and so

$$
\begin{aligned}
& \|P(u)-P(\widetilde{u})\| \\
& \leq\left[\psi(1)+\left\|\psi^{\prime}\right\|_{L^{1}(0,1 ; \mathbb{R})}+\ell_{1}\|\psi\|_{L^{1}(0,1 ; \mathbb{R})}\right]\|u-\widetilde{u}\|_{C([-\tau, 1] ; X)} .
\end{aligned}
$$

On the other hand, we have

$$
\|F(u)-F(\widetilde{u})\| \leq\|J(u) P(u)-J(\widetilde{u}) P(u)\|+\|J(\widetilde{u}) P(u)-J(\widetilde{u}) P(\widetilde{u})\| .
$$

Since

$$
\|u\|_{C([-\tau, 1] ; X)} \leq\|h\|_{C([-\tau, 0] ; X)}+\|u\|_{C([0,1] ; X)},
$$

from $(2.10),(2.11),(2.12)$ and $(2.13)$, we get

$$
\begin{aligned}
& \|F(u)-F(\widetilde{u})\| \leq \frac{\ell\|\psi\|_{L^{1}(0,1 ; \mathbb{R})}}{m^{2}}\|P(u)\|\|u-\widetilde{u}\|_{C([0,1] ; X)}+\frac{1}{m}\|P(u)-P(\widetilde{u})\| \\
& \leq \frac{\ell\|\psi\|_{L^{1}(0,1 ; \mathbb{R})}}{m^{2}}\left[k_{0}+k_{1}\|u\|_{C([0,1] ; X)}\right]\|u-\widetilde{u}\|_{C([0,1] ; X)} \\
& +\frac{1}{m}\left[\psi(1)+\left\|\psi^{\prime}\right\|_{L^{1}(0,1 ; \mathbb{R})}+\ell_{1}\|\psi\|_{L^{1}(0,1 ; \mathbb{R})}\right]\|u-\widetilde{u}\|_{C([0,1] ; X)},
\end{aligned}
$$

where (we recall that, by $(2.6),\|\eta\|=\|A \xi+\psi(0) h(0)\|$ )

$$
\left\{\begin{array}{l}
k_{0}:=\|A \xi+\psi(0) h(0)\|+\left[\ell_{2}+\ell_{1}\|h\|_{C([-\tau, 0] ; X)}\right]\|\psi\|_{L^{1}(0,1 ; \mathbb{R})}, \\
k_{1}:=\psi(1)+\left\|\psi^{\prime}\right\|_{L^{1}(0,1 ; \mathbb{R})}+\ell_{1}\|\psi\|_{L^{1}(0,1 ; \mathbb{R})} .
\end{array}\right.
$$

So, if the set $K \subseteq C_{[h]}([-\tau, 0] ; X)$ is bounded, say by $r>0$, we conclude that

$$
\|F(u)-F(\widetilde{u})\| \leq L\|u-\widetilde{u}\|_{C([0,1] ; X)},
$$


for each $u, \widetilde{u} \in K$, where

$$
L:=\frac{\ell\|\psi\|_{L^{1}(0,1 ; \mathbb{R})}}{m^{2}}\left(k_{0}+k_{1} r\right)+\frac{1}{m}\left[\psi(1)+\left\|\psi^{\prime}\right\|_{L^{1}(0,1 ; \mathbb{R})}+\ell_{1}\|\psi\|_{L^{1}(0,1 ; \mathbb{R})}\right] .
$$

Finally, from Remark 2.2 and (2.12), we deduce that

$$
\|F(u)\| \leq L_{0}\|u\|_{C([-\tau, 1] ; X)}+M_{0}
$$

for each $u \in C_{[h]}([-\tau, 1] ; X)$, with

$$
L_{0}:=\frac{k_{1}}{m}, \quad M_{0}:=\frac{k_{2}}{m},
$$

where $k_{1}$ is given by (2.14) and

$$
k_{2}:=\|A \xi+\psi(0) h(0)\|+\ell_{2}\|\psi\|_{L^{1}(0,1 ; \mathbb{R})} .
$$

Thus $F$ satisfies the linear growth condition (2.9) and this completes the proof.

\section{The main result}

Theorem 3.1. Let the assumptions $\left(H_{A}\right),\left(H_{\varphi}\right),\left(H_{f}\right)$ and $\left(H_{\psi}\right)$ be satisfied. Let us assume that

$$
m_{2} L_{0}+\ell_{1}<\omega
$$

where $L_{0}>0$ is given by (2.15). Let $h \in C([-\tau, 0] ; X)$ with $h(0) \in D(A)$. Then the identification problem (JP) has at least one mild solution $(z, u)$, where $u$ is a Lipschitz continuous mild solution of the direct problem

$$
\begin{cases}u^{\prime}(t)=A u(t)+\varphi\left(t, u_{t}\right) F(u)+f\left(t, u_{t}\right), & t \in[0,1], \\ u(t)=h(t), & t \in[-\tau, 0],\end{cases}
$$

and

$$
z=F(u)
$$

$F$ being defined by (2.5) and (2.6). 
Remark 3.1. Condition (3.1) says that $\omega$ is large if compared with the Lipschitz constant of $f$, the $L^{1}$-norms of $\psi$ and $\psi^{\prime}$ and the value of $\psi$ at 1 . See the definition of $L_{0}$, i.e., (2.15) and (2.14).

For instance, if $\psi \equiv 1$, i.e., if $\psi(t) d t$ is the Lebesgue measure, then, according to Remark 2.2, (3.1) is satisfied if

$$
\frac{m_{2}}{m_{1}}\left(1+\ell_{1}\right)+\ell_{1}<\omega
$$

where $\omega>0, m_{1}>0, m_{2}>0$ and $\ell_{1}>0$ are defined in the hypotheses $\left(H_{A}\right),\left(H_{\varphi}\right)$ and respectively $\left(H_{f}\right)$. If $(3.1)$ is satisfied then, for each $h \in$ $C([-\tau, 0] ; X)$, there exists $r_{h}>0$ such that

$$
4\|h\|_{C([-\tau, 0] ; X)}+\frac{m_{2}\left(L_{0} r+M_{0}\right)+\ell_{2}}{\omega-\ell_{1}} \leq r
$$

for each $r \geq r_{h}$, where $L_{0}>0, M_{0}>0$ are given by (2.15) and $\ell_{2}>0$ by $\left(H_{f}\right)$.

\subsection{The idea of the proof}

It is easy to observe that (JP) has a mild (strict) solution if and only if (3.2) has a mild (strict) solution $u$ and $z$ is given by (3.3).

Let $h \in C([-\tau, 0] ; X)$ with $h(0) \in D(A)$ and $\xi \in D(A)$ be arbitrary but fixed, let $r>\max \left\{0,\|h\|_{C([-\tau, 0] ; X)}\right\}$ and, for any $\mathcal{L}>0$, let us define the sets

$C_{\mathcal{L}}([-\tau, 1] ; X):=\{u \in C([-\tau, 1] ; X) ;\|u(t)-u(s)\| \leq \mathcal{L}|t-s|, \forall t, s \in[0,1]\}$

and

$$
\mathcal{K}:=\left\{u \in C_{[h]}([-\tau, 1] ; X) \cap C_{\mathcal{L}}([-\tau, 1] ; X) ;\|u\|_{C([-\tau, 1] ; X)} \leq r\right\},
$$

$C_{[h]}([-\tau, 1] ; X)$ being defined by $(2.8)$. Clearly $\mathcal{K}$ is nonempty, closed and convex in $C([-\tau, 1] ; X)$. Let us define the operator $\mathcal{S}: \mathcal{K} \rightarrow C([-\tau, 1] ; X)$ by

$$
\mathcal{S}(x):=u,
$$

where $u \in C([-\tau, 1] ; X)$ is the unique mild solution of the semilinear delay evolution problem

$$
\begin{cases}u^{\prime}(t)=A u(t)+\varphi\left(t, u_{t}\right) F(x)+f\left(t, u_{t}\right), & t \in[0,1] \\ u(t)=h(t), & t \in[-\tau, 0] .\end{cases}
$$


We will show that, under the hypotheses of Theorem 3.1, we can choose $r>0$ and $\mathcal{L}>0$ such that the operator $\mathcal{S}$ maps $\mathcal{K}$ into itself, is continuous and $\mathcal{S}(\mathcal{K})$ is relatively compact in $C([-\tau, 1] ; X)$. Then, by Schauder's Theorem, $\mathcal{S}$ has at least one fixed point $u \in \mathcal{K}$, which provides a mild solution $(z, u)$ for $(\mathcal{J P})$, where $z=F(u)$.

\section{Proof of the main result}

The Bellman-type inequality below is a variant of Lemma 4.3 in BURLICA and Roşu [2]. Since its proof is almost identical with the one of Lemma 4.3 in BuRlic $\breve{A}$ and Roşu [2], we do not give details.

Lemma 4.1. Let $T>0$ and let $y:[-\tau, T] \rightarrow \mathbb{R}_{+}$and $\alpha_{0}, \beta:[0, T] \rightarrow$ $\mathbb{R}_{+}$be continuous functions, $\alpha_{0}$ being nondecreasing. If

$$
y(t) \leq \alpha_{0}(t)+\int_{0}^{t} \beta(s)\left\|y_{s}\right\|_{C([-\tau, 0]: \mathbb{R})} d s
$$

for each $t \in[0, T]$, then

$$
y(t) \leq \alpha(t)+\int_{0}^{t} \alpha(s) \beta(s) \exp \left(\int_{s}^{t} \beta(\sigma) d \sigma\right) d s,
$$

for each $t \in[0, T]$, where

$$
\alpha(t):=\left\|y_{0}\right\|_{C([-\tau, 0] ; \mathbb{R})}+\alpha_{0}(t)
$$

for each $t \in[0, T]$.

We will show how to choose $\mathcal{L}>0$ such that the operator $\mathcal{S}$, defined as above, maps $\mathcal{K}$ given by (3.5) into itself. More precisely, we will prove

Lemma 4.2. Let $\left(H_{A}\right),\left(H_{\varphi}\right),\left(H_{f}\right)$ and $\left(H_{\psi}\right)$ be satisfied. Let $h \in$ $C^{1}([-\tau, 0] ; X)$ with $h(0) \in D(A)$ and let us assume that (3.1) hold. Then, there exist $r>0$ and $\mathcal{L}>0$ such that the operator $\mathcal{S}$, defined by (3.6), maps the set $\mathcal{K}$ defined by (3.5) into itself, is continuous and $\mathcal{S}(\mathcal{K})$ is compact.

Proof. Let $u$ be the unique mild solution of the problem (3.7). Since, by $\left(A_{2}\right)$ in $\left(H_{A}\right), A$ generates a contraction semigroup $\{S(t) ; t \geq 0\}$ satisfying $\|S(t)\| \leq e^{-\omega t}$ for each $t \geq 0$, using the variation of constants formula, $\left(\varphi_{2}\right)$ in $H_{\varphi}$ and $\left(H_{f}\right)$, we deduce

$$
\|u(t)\| \leq e^{-\omega t}\|u(0)\|+\int_{0}^{t} e^{-\omega(t-s)}\left[m_{2}\|F(x)\|+\ell_{1}\left\|u_{s}\right\|_{C([-\tau, 0] ; \mathbb{R})}+\ell_{2}\right] d s .
$$


Whence

$$
e^{\omega t}\|u(t)\| \leq\|u(0)\|+\int_{0}^{t} e^{\omega s}\left[m_{2}\|F(x)\|+\ell_{1}\left\|u_{s}\right\|_{C([-\tau, 0] ; \mathbb{R})}+\ell_{2}\right] d s .
$$

Let us fix an $r>\|h\|_{C([-\tau, 0] ; X)}$ which will be chosen more precisely later on. By virtue of $(2.9),\|F(x)\| \leq L_{0}\|x\|_{C([-\tau, 1] ; X)}+M_{0} \leq L_{0} r+M_{0}$, for each $x \in C_{[h]}([-\tau, 1] ; X)$ with $\|x\|_{C([-\tau, 1] ; X)} \leq r$, where $L_{0}$ and $M_{0}$ are given by $(2.15)$. Denoting by

$$
k=m_{2}\left(L_{0} r+M_{0}\right)+\ell_{2},
$$

the preceding inequality can be rewritten as

$$
e^{\omega t}\|u(t)\| \leq\|u(0)\|+\frac{k}{\omega}\left(e^{\omega t}-1\right)+\int_{0}^{t} \ell_{1} e^{\omega s}\left\|u_{s}\right\|_{C([-\tau, 0] ; \mathbb{R})} d s
$$

for each $t \in[0,1]$. But this inequality is of the form (4.1) for the specific choice of $y, \alpha_{0}$, and $\beta$ as specified below:

$$
\begin{cases}y(t)=e^{\omega t}\|u(t)\|, & t \in[-\tau, 1] \\ \alpha_{0}(s)=\|u(0)\|+\frac{k}{\omega}\left(e^{\omega s}-1\right), & s \in[0,1] \\ \beta(s)=\ell_{1}, & s \in[0,1] .\end{cases}
$$

Observing that

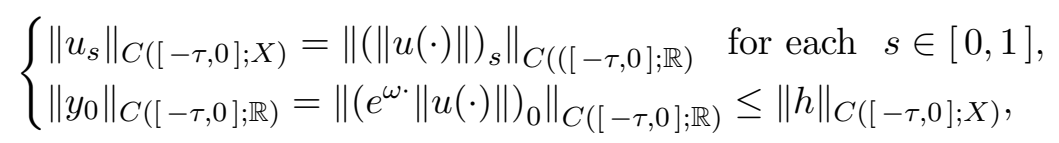

from Lemma 4.1, we deduce that the function $y$, defined as before, satisfies (4.2). Equivalently, $t \mapsto\|u(t)\|$ satisfies

$$
\|u(t)\| \leq e^{-\omega t} \alpha(t)+e^{-\omega t} \int_{0}^{t} \alpha(s) \beta(s) \exp \left(\int_{s}^{t} \beta(\sigma) d \sigma\right) d s
$$

for each $t \in[0,1]$. Recalling the definition of $\alpha, \beta$ - see Lemma 4.1 - and taking into account that $\|u(0)\|=\|h(0)\| \leq\|h\|_{C([-\tau, 0] ; X)}$ we conclude that

$$
\begin{aligned}
\|u(t)\| & \leq e^{-\omega t}\left[2\|h\|_{C([-\tau, 0] ; X)}+\frac{k}{\omega}\left(e^{\omega t}-1\right)\right] \\
& +e^{-\omega t} \int_{0}^{t}\left[2\|h\|_{C([-\tau, 0] ; X)}+\frac{k}{\omega}\left(e^{\omega s}-1\right)\right] \ell_{1} e^{\ell_{1}(t-s)} d s
\end{aligned}
$$


for each $t \in[0,1]$.

Since, by (3.1), $\omega-\ell_{1}>0$, the second term on the right-hand side of the preceding inequality can be estimated as

$$
\begin{aligned}
& e^{-\omega t} \int_{0}^{t}\left[2\|h\|_{C([-\tau, 0] ; X)}+\frac{k}{\omega}\left(e^{\omega s}-1\right)\right] \ell_{1} e^{\ell_{1}(t-s)} d s \\
& \leq \ell_{1} e^{-\left(\omega-\ell_{1}\right) t} \int_{0}^{t}\left[2\|h\|_{C([-\tau, 0] ; X)}+\frac{k}{\omega} e^{\omega s}\right] e^{-\ell_{1} s} d s \\
& =2 \ell_{1} e^{-\left(\omega-\ell_{1}\right) t} \frac{1-e^{-\ell_{1} t}}{\ell_{1}}\|h\|_{C([-\tau, 0] ; X)}+\ell_{1} \frac{k\left[1-e^{-\left(\omega-\ell_{1}\right) t}\right]}{\omega\left(\omega-\ell_{1}\right)} \\
& \leq 2\|h\|_{C([-\tau, 0] ; \mathbb{R})}+\frac{\ell_{1} k}{\omega\left(\omega-\ell_{1}\right)} .
\end{aligned}
$$

From (4.3) and the last relation, taking into account that, for $t \geq 0$,

$$
e^{-\left(\omega-\ell_{1}\right) t}\left(1-e^{-\ell_{1} t}\right) \leq 1, \quad 1-e^{-\left(\omega-\ell_{1}\right) t} \leq 1
$$

we get

$$
\|u(t)\| \leq 4\|h\|_{C([-\tau, 0] ; \mathbb{R})}+\frac{k}{\omega-\ell_{1}}
$$

for each $t \in[0,1]$, Now, recalling that $k=m_{2}\left(L_{0} r+M_{0}\right)+\ell_{2}$, we conclude that

$$
\|u(t)\| \leq 4\|h\|_{C([-\tau, 0] ; \mathbb{R})}+\frac{m_{2}\left(L_{0} r+M_{0}\right)+\ell_{2}}{\omega-\ell_{1}}
$$

for each $t \in[-\tau, 1]$. From (3.1) and the last part of Remark 3.1, it follows that there exists $r_{h}>0$ such that (3.4) holds for each $r \geq r_{h}$.

Let us fix $r \geq \max \left\{r_{h},\|h\|_{C([-\tau, 0] ; X)}\right\}$. From (4.4), we deduce that for each $x \in C_{[h]}([-\tau, 1] ; X)$, satisfying

$$
\|x\|_{C_{[h]}([-\tau, 1] ; X)} \leq r
$$

we have

$$
\|u\|_{C([\tau, 1] ; X)} \leq r
$$

which shows that $\mathcal{S}$ maps the set $\left\{x \in C_{[h]}([-\tau, 1] ; X) ;\|x\|_{C_{[h]}([-\tau, 1] ; X)} \leq r\right\}$ into itself. 
Next, we show how to choose the Lipschitz constant $\mathcal{L}>0$ such that the set $\mathcal{K}$, defined by means of $(3.5)$, is invariant under $\mathcal{S}, \mathcal{S}(\mathcal{K})$ is relatively compact in $C_{[h]}([-\tau, 1] ; X)$ and $\mathcal{S}$ is continuous from $\mathcal{K}$ into $\mathcal{K}$. To this aim, let us observe that, for each $x \in C_{[h]}([-\tau, 1] ; X)$, we have

$$
u(t)=S(t) u(0)+\int_{0}^{t} \varphi\left(s, u_{s}\right) S(t-s) F(x) d s+\int_{0}^{t} S(t-s) f\left(s, u_{s}\right) d s
$$

and

$$
\begin{aligned}
u(t+\theta) & =S(t) u(\theta)+\int_{\theta}^{t+\theta} \varphi\left(s, u_{s}\right) S(t+\theta-s) F(x) d s \\
& +\int_{\theta}^{t+\theta} S(t+\theta-s) f\left(s, u_{s}\right) d s \\
& =S(t) u(\theta)+\int_{0}^{t} \varphi\left(s+\theta, u_{s+\theta}\right) S(t-s) F(x) d s \\
& +\int_{0}^{t} S(t-s) f\left(s+\theta, u_{s+\theta}\right) d s .
\end{aligned}
$$

So, we get

$$
\begin{aligned}
& \|u(t+\theta)-u(t)\| \leq e^{-\omega t}\|u(\theta)-u(0)\| \\
& +\ell \int_{0}^{t} e^{-\omega(t-s)}\left[\theta+\left\|u_{s+\theta}-u_{s}\right\|_{C([-\tau, 0] ; X)}\right]\|F(x)\| d s \\
& +\ell_{1} \int_{0}^{t} e^{-\omega(t-s)}\left[\theta+\left\|u_{s+\theta}-u_{s}\right\|_{C([-\tau, 0] ; X)}\right]\|F(x)\| d s
\end{aligned}
$$

for each $\theta \in(0,1)$ and $t \in[0,1-\theta]$. Whence

$$
\begin{aligned}
& \|u(t+\theta)-u(t)\| \leq e^{-\omega t}\|u(\theta)-u(0)\|+\frac{\theta}{\omega}\left(1-e^{-\omega t}\right)\left[\ell\|F(x)\|+\ell_{1}\right] \\
& +\left[\ell\|F(x)\|+\ell_{1}\right] \int_{0}^{t} e^{-\omega(t-s)}\left\|u_{s+\theta}-u_{s}\right\|_{C([-\tau, 0] ; X)} d s .
\end{aligned}
$$

Thanks to (2.9), we have

$$
\|F(x)\| \leq L_{0}\|x\|_{C([-\tau, 1] ; X)}+M_{0} \leq L_{0} r+M_{0},
$$

for each $x \in C_{[h]}([-\tau, 1] ; X)$ with $\|x\|_{C([-\tau, 1] ; X)} \leq r$, where $L_{0}$ and $M_{0}$ are given by (2.15). So, setting

$$
\eta:=\ell\left(L_{0} r+M_{0}\right)+\ell_{1},
$$


the above inequality leads to

$$
\begin{aligned}
e^{\omega t}\|u(t+\theta)-u(t)\| & \leq\|u(\theta)-u(0)\|+\frac{\eta}{\omega}\left(e^{\omega t}-1\right) \theta \\
& +\eta \int_{0}^{t} e^{\omega s}\left\|u_{s+\theta}-u_{s}\right\|_{C([-\tau, 0] ; X)} d s
\end{aligned}
$$

for each $\theta \in(0,1)$ and $t \in[0,1-\theta]$. Clearly, (4.9) is of the form (4.1), with

$$
\begin{cases}y(t)=e^{\omega t}\|u(t+\theta)-u(t)\|, & t \in[-\tau, 1-\theta], \\ \alpha_{0}(s)=\|u(\theta)-u(0)\|+\frac{\eta}{\omega}\left(e^{\omega s}-1\right) \theta, & s \in[0,1-\theta], \\ \beta(s)=\eta, & s \in[0,1-\theta] .\end{cases}
$$

Thanks to Lemma 4.1, the function $y$, defined above, satisfies (4.2) on $[0,1-\theta]$ with $\alpha$ given by

$$
\alpha(s)=\|u(\theta)-u(0)\|+\frac{\eta}{\omega}\left(e^{\omega s}-1\right) \theta+\left\|u_{\theta}-u_{0}\right\|_{C([-\tau, 0] ; X)} .
$$

Equivalently, the function $t \mapsto\|u(t+\theta)-u(t)\|$ satisfies

$$
\|u(t+\theta)-u(t)\| \leq e^{-\omega t} \alpha(t)+e^{-\omega t} \eta \int_{0}^{t} \alpha(s) e^{\eta(t-s)} d s
$$

for $t \in[0,1-\theta]$. Obviously

$$
\alpha(s) \leq 2\left\|u_{\theta}-u_{0}\right\|_{C([-\tau, 0] ; X)}+\frac{\eta}{\omega} e^{\omega s} \theta
$$

and therefore

$$
\begin{aligned}
\|u(t+\theta)-u(t)\| & \leq e^{-\omega t}\left[2\left\|u_{\theta}-u_{0}\right\|_{C([-\tau, 0] ; X)}+\frac{\eta}{\omega} e^{\omega t} \theta\right] \\
& +e^{-\omega t} \eta \int_{0}^{t}\left[2\left\|u_{\theta}-u_{0}\right\|_{C([-\tau, 0] ; X)}+\frac{\eta}{\omega} e^{\omega s} \theta\right] e^{\eta(t-s)} d s .
\end{aligned}
$$

Estimating the second term on the right-hand side, we get

$$
\begin{aligned}
& e^{-\omega t} \eta \int_{0}^{t}\left[2\left\|u_{\theta}-u_{0}\right\|_{C([-\tau, 0] ; X)}+\frac{\eta}{\omega} e^{\omega s} \theta\right] e^{\eta(t-s)} d s \\
& =e^{-(\omega-\eta) t} \eta \int_{0}^{t}\left[2\left\|u_{\theta}-u_{0}\right\|_{C([-\tau, 0] ; X)}+\frac{\eta}{\omega} e^{\omega s} \theta\right] e^{-\eta s} d s \\
& =e^{-(\omega-\eta) t} \eta\left\{\frac{1-e^{-\eta t}}{\eta} 2\left\|u_{\theta}-u_{0}\right\|_{C([-\tau, 0] ; X)}+\frac{\eta\left[e^{(\omega-\eta) t}-1\right]}{\omega(\omega-\eta)} \theta\right\} \\
& \leq 2 e^{-(\omega-\eta) t}\left\|u_{\theta}-u_{0}\right\|_{C([-\tau, 0] ; X)}+\frac{\eta^{2}\left[1-e^{-(\omega-\eta) t}\right]}{\omega(\omega-\eta)} \theta .
\end{aligned}
$$


Let

$$
\mu=\sup \left\{e^{-(\omega-\eta) t} ; t \in[0,1]\right\} .
$$

Then, we have both

$$
e^{-(\omega-\eta) t} \leq \mu, \text { and } \frac{1-e^{-(\omega-\eta) t}}{\omega-\eta} \leq \mu .
$$

To prove the last inequality, first, let us observe that we may assume with no loss of generality that $\omega-\eta \neq 0$. Indeed, if either $\omega>\eta$ or $\omega<\eta$, we have nothing to do. If $\omega=\eta$, then we can slightly diminish $\omega>0$ such that both (3.1) and $\omega-\eta \neq 0$ hold true. Next, by Lagrange MeanValue Theorem applied to the function $x \mapsto e^{-x}$ either on $[0,-(\omega-\eta) t]$ if $\omega-\eta<0$ or on $[-(\omega-\eta) t, 0]$ if $\omega-\eta>0$, it follows that there exists $t_{1} \in[0,1]$ such that, in both cases mentioned before, we have

$$
\frac{1-e^{-(\omega-\eta) t}}{\omega-\eta}=\frac{e^{-(\omega-\eta) t_{1}}}{\omega-\eta}(\omega-\eta) t=e^{-(\omega-\eta) t_{1}} t \leq e^{-(\omega-\eta) t_{1}} \leq \mu .
$$

Accordingly, we get

$$
\begin{aligned}
& e^{-\omega t} \eta \int_{0}^{t}\left[2\left\|u_{\theta}-u_{0}\right\|_{C([-\tau, 0] ; X)}+\frac{\eta}{\omega} e^{\omega s} \theta\right] e^{\eta(t-s)} d s \\
& \leq 2 \mu\left\|u_{\theta}-u_{0}\right\|_{C([-\tau, 0] ; X)}+\frac{\eta^{2} \mu}{\omega} \theta
\end{aligned}
$$

Finally, we obtain

$$
\begin{aligned}
\|u(t+\theta)-u(t)\| & \leq 2\left\|u_{\theta}-u_{0}\right\|_{C([-\tau, 0] ; X)}+\frac{\eta}{\omega} \theta \\
& +2 \mu\left\|u_{\theta}-u_{0}\right\|_{C([-\tau, 0] ; X)}+\frac{\eta^{2} \mu}{\omega} \theta
\end{aligned}
$$

and thus

$$
\|u(t+\theta)-u(t)\| \leq 2(1+\mu)\left\|u_{\theta}-u_{0}\right\|_{C([-\tau, 0] ; X)}+\frac{\eta(1+\eta \mu)}{\omega} \theta,
$$

for each $\theta \in(0,1)$ and $t \in[0,1-\theta]$.

We estimate now

$$
\left\|u_{\theta}-u_{0}\right\|_{C([-\tau, 0] ; X)}=\sup _{t \in[-\tau, 0]}\|u(t+\theta)-u(t)\| .
$$


Let $t \in[-\tau, 0]$ and $\theta \in(0,1)$. We have two complementary cases.

Case 1. $-\tau \leq t+\theta \leq 0$. Since $h \in C^{1}([-\tau, 0] ; X)$, we have

$$
\|u(t+\theta)-u(t)\| \leq\|h(\cdot+\theta)-h(\cdot)\|_{C([-\tau, 0] ; X)} \leq\left\|h^{\prime}\right\|_{C([-\tau, 0] ; X)} \theta .
$$

Case 2. $-\tau \leq t \leq 0<t+\theta$. Then we get

$$
\|u(t+\theta)-u(t)\|=\|u(t+\theta)-h(t)\| \leq\|u(t+\theta)-u(0)\|+\|h(0)-h(t)\| .
$$

At this point, since $0<t+\theta<\theta$, from (4.7) and (2.9), we deduce

$$
\begin{aligned}
\|u(t+\theta)-u(0)\| & \leq\|S(t+\theta) u(0)-u(0)\| \\
& +\int_{0}^{t+\theta}\left[\varphi\left(s, u_{s}\right)\|F(x)\|+\left\|f\left(s, u_{s}\right)\right\|\right] d s \\
& \leq\left[\|A h(0)\|+m_{2}\left(L_{0} r+M_{0}\right)+\ell_{1} r+\ell_{2}\right](t+\theta) \\
& \leq\left[\|A h(0)\|+m_{2}\left(L_{0} r+M_{0}\right)+\ell_{1} r+\ell_{2}\right] \theta .
\end{aligned}
$$

Further, since $-\theta<t \leq 0$, we have $|t|<\theta$ and thus

$$
\|h(0)-h(t)\| \leq\left\|h^{\prime}\right\|_{C([-\tau, 0],] ; X)}|t| \leq\left\|h^{\prime}\right\|_{C([-\tau, 0],] ; X)} \theta .
$$

Summing up, from (4.12), (4.13) and (4.14), we get

$$
\|u(t+\theta)-u(t)\| \leq\left[\|A h(0)\|+m_{2}\left(L_{0} r+M_{0}\right)+\ell_{1} r+\ell_{2}+\left\|h^{\prime}\right\|_{C([-\tau, 0] ; X)}\right] \theta
$$

for each $\theta \in(0,1)$ and $t \in[0,1-\theta]$. So, we have proved the estimate

$$
\|u(\cdot+\theta)-u\|_{C([-\tau, 0] ; X} \leq \mathcal{L} \theta
$$

for each $x \in C_{[h]}([-\tau, 1] ; X)$ with $\|x\|_{C([-\tau, 1] ; X)} \leq r$, each $t \in[0,1]$ and for each $\theta \in(0,1)$ and $t \in[0,1-\theta]$, where $\mathcal{L}$ is given by

$$
\begin{aligned}
\mathcal{L} & =2(1+\mu)\left[\|A h(0)\|+m_{2}\left(L_{0} r+M_{0}\right)+\ell_{1} r+\ell_{2}+\left\|h^{\prime}\right\|_{C([-\tau, 0] ; X)}\right] \\
& +\frac{\eta(1+\eta \mu)}{\omega}
\end{aligned}
$$

$\eta$ being defined by (4.8) and $\mu$ by (4.10). Let us define $\mathcal{K}$ as in (3.5), i.e.,

$$
\mathcal{K}:=\left\{x \in C_{[h]}([-\tau, 1] ; X) \cap C_{\mathcal{L}}([-\tau, 1] ; X) ;\|u\|_{C([-\tau, 1] ; X)} \leq r\right\}
$$

where $r>0$ and $\mathcal{L}>0$ are chosen as above and $C_{\mathcal{L}}([-\tau, 1] ; X):=\{u \in C([-\tau, 1] ; X) ;\|u(t)-u(s)\| \leq \mathcal{L}|t-s|, \forall t, s \in[0,1]\}$. 
From (4.6) and (4.15), we conclude that $\mathcal{S}$ maps $\mathcal{K}$ into itself.

To prove the relative compactness of $\mathcal{S}(\mathcal{K})$ in $C([-\tau, 1] ; X)$, let us note that, thanks to the fact that $u(t)=h(t)$ for each $u \in \mathcal{K}$ and each $t \in[-\tau, 0]$, it suffices to show that $\mathcal{K}$ is relatively compact in $C([0,1] ; X)$. Clearly $\mathcal{S}(\mathcal{K})$ is equicontinuous from the right on $[0,1)$, containing only Lipschitz functions with the very same constant $\mathcal{L}$. Furthermore, the set

$$
\left\{t \mapsto \varphi\left(t, u_{t}\right) F(x)+f\left(t, u_{t}\right) ; x \in \mathcal{K}, u=\mathcal{S}(x)\right\}
$$

is uniformly bounded on $[0,1]$ and so Lebesgue uniformly integrable in $L^{1}(0,1 ; X)$. Thus, $\mathcal{S}(\mathcal{K})$ satisfies all the hypotheses of " $\left(c_{1}\right)$ implies $\left(c_{2}\right)$ " part in Theorem 2.1. Whence $\mathcal{S}(\mathcal{K})$ is relatively compact in $C([0,1] ; X)$.

Finally, let $\left(x_{p}\right)_{p \in \mathbb{N}}$ is a sequence in $\mathcal{K}$ which converges uniformly to $x \in \mathcal{K}$. As $F$ is Lipschitz continuous, it follows that

$$
\lim _{p} F\left(x_{p}\right)=F(x)
$$

Now, if some subsequence of $\left(\mathcal{S}\left(x_{p}\right)\right)_{p \in \mathbb{N}}$ converges to a certain function $u$, recalling that $\varphi, F$ and $f$ are jointly continuous, it follows that $u$ must coincide with $\mathcal{S}(x)$. But $\left\{\mathcal{S}\left(x_{p}\right) ; p \in \mathbb{N}\right\}$ is relatively compact in $C([0,1] ; X)$. Using this remark and a usual argument by contradiction, we deduce that the sequence itself converges to $\mathcal{S}(x)$. So $\mathcal{S}$ is continuous and this completes the proof of Lemma 4.2.

Now, we can pass to the proof of Theorem 3.1.

Proof. By Lemma 4.2 and Schauder's Fixed Point Theorem, we conclude that the operator $\mathcal{S}$, defined by (3.6), has at least one fixed point $u \in \mathcal{K}$. But each fixed point of $\mathcal{S}$ is a mild solution of the problem (3.2) which, by the definition of $\mathcal{K}$, is Lipschitz continuous with constant $\mathcal{L}$. Obviously $u$ is a generalized solution of $(\mathcal{J P})$. Since $A$ satisfies $\left(A_{2}\right)$, by virtue of $(i)$ in Remark 2.2 combined with Lemma 2.2, it follows that $(z, u)$, with $z$ given by $(3.3)$, is a mild solution of $(\mathcal{J P})$ and this completes the proof of Theorem 3.1.

\section{Uniqueness and continuous dependence}

The aim of this Section is to state and prove a uniqueness and continuous dependence result referring to mild solutions of $(\mathcal{J P})$ in the sense of Definition 2.2. First, some preliminary evaluations are needed. As $u$ satisfies 
(2.7), where $F$ depends on $u$ but also on $\xi$ and $h$, we can express $z$ in terms of $u, \xi, h$ as:

$$
z=F(u, \xi, h),
$$

where $F(u, \xi, h)$ is defined (cf. (2.4), (2.5) and (2.6)) by

$F(u, \xi, h)=J(u, h)\left[\eta(\xi, h)+\psi(1) u(1)-\int_{0}^{1} \psi^{\prime}(t) u(t) d t-\int_{0}^{1} \psi(t) f\left(t, u_{t}\right) d t\right]$,

$\eta(\xi, h)=-A \xi-\psi(0) h(0)$,

$J(u, h)=\left[\int_{0}^{1} \psi(t) \varphi\left(t, u_{t}\right) d t\right]^{-1}$.

Recall now that, by $($ ii $)$ in Remark 2.2 , we have

$$
J(u, h) \leq m^{-1}
$$

for all $u \in C([0,1] ; X)$ and $h \in C([-\tau, 0] ; X)$.

Let $(\xi, h),(\widetilde{\xi}, \widetilde{h}) \in D(A) \times C([-\tau, 0] ; X)$ be such that $h(0), \widetilde{h}(0) \in D(A)$ and let $u$ and $\widetilde{u}$ be two mild solutions of $(\mathcal{J P})$, the first one corresponding to $(\xi, h)$ and the second one to $(\widetilde{\xi}, \widetilde{h})$. We emphasize that, for the time being, we do not know that $u$ and $\widetilde{u}$ are uniquely determined. Let us observe that, in order to prove both the uniqueness and the continuous dependence of $u$ on $(\xi, h)$, we need some sharp evaluations of the difference $u-\widetilde{u}$. To obtain this, we have first to evaluate $|J(u, h)-J(\widetilde{u}, \widetilde{h})|$ and $\|F(u, \xi, h)-F(\widetilde{u}, \widetilde{\xi}, \widetilde{h})\|$.

\subsection{An estimate of $F(u, \xi, h)-F(\widetilde{u}, \widetilde{\xi}, \widetilde{h})$}

First, we observe that, for each $h, \widetilde{h} \in C([-\tau, 0] ; X), u \in C_{[h]}([-\tau, 1] ; X)$, $\widetilde{u} \in C_{\widetilde{[h}]}([-\tau, 1] ; X)$ and each $t \in[0,1]$, we have

$$
\begin{aligned}
\left\|u_{t}-\widetilde{u}_{t}\right\|_{C([-\tau, 0] ; X)} & =\sup _{s \in[-\tau, 0]}\|u(t+s)-\widetilde{u}(t+s)\| \\
& \leq \sup _{\widetilde{t} \in[0,1]} \sup _{s \in[-\tau, 0]}\|u(\widetilde{t}+s)-\widetilde{u}(\widetilde{t}+s)\| \\
& =\max \left\{\|u-\widetilde{u}\|_{C([0,1] ; X)},\|h-\widetilde{h}\|_{C([-\tau, 0] ; X)}\right\} \\
& \leq\|u-\widetilde{u}\|_{C([0,1] ; X)}+\|h-\widetilde{h}\|_{C([-\tau, 0] ; X)} .
\end{aligned}
$$


Whence, from (5.1), $\left(\varphi_{1}\right)$ in $\left(H_{\varphi}\right)$ and $\left(f_{1}\right)$ in $\left(H_{f}\right)$ (cf. Subsection 2.3), we deduce

$$
\begin{aligned}
& |J(u, h)-J(\widetilde{u}, \widetilde{h})| \leq m^{-2} \int_{0}^{1} \psi(t)\left|\varphi\left(t, u_{t}\right)-\varphi\left(t, \widetilde{u}_{t}\right)\right| d t \\
& \leq \ell m^{-2} \int_{0}^{1} \psi(t)\left\|u_{t}-\widetilde{u}_{t}\right\|_{C([-\tau, 0] ; X)} d t \\
& \leq \ell m^{-2}\|\psi\|_{L^{1}(0,1 ; \mathbb{R})}\left[\|u-\widetilde{u}\|_{C([0,1] ; X)}+\|h-\widetilde{h}\|_{C([-\tau, 0] ; X)}\right] d t
\end{aligned}
$$

for all $h, \widetilde{h} \in C([-\tau, 0] ; X), u \in C_{[h]}([-\tau, 1] ; X)$ and $\widetilde{u} \in C_{\widetilde{h}]}([-\tau, 1] ; X)$.

Likewise

$$
\begin{aligned}
& \left\|\int_{0}^{1} \psi(t)\left[f\left(t, u_{t}\right)-f\left(t, \widetilde{u}_{t}\right)\right] d t\right\| \\
& \leq \ell_{1}\|\psi\|_{L^{1}(0,1 ; \mathbb{R})}\left[\|u-\widetilde{u}\|_{C([0,1] ; X)}+\|h-\widetilde{h}\|_{C([-\tau, 0] ; X)}\right] d t
\end{aligned}
$$

for all $h, \widetilde{h} \in C([-\tau, 0] ; X), u \in C_{[h]}([-\tau, 1] ; X)$ and $\widetilde{u} \in C_{\widetilde{[h]}}([-\tau, 1] ; X)$. Then, we get

$$
\begin{aligned}
& \|F(u, \xi, h)-F(\widetilde{u}, \widetilde{\xi}, \widetilde{h})\| \leq|J(u, h)-J(\widetilde{u}, \widetilde{h})|[\|\eta(\xi, h)\|+\psi(1)\|u(1)\| \\
& \left.+\int_{0}^{1}\left|\psi^{\prime}(t)\right|\|u(t)\| d t+\int_{0}^{1} \psi(t)\left\|f\left(t, u_{t}\right)\right\| d t\right] \\
& +|J(\widetilde{u}, \widetilde{h})|[\|\eta(\xi-\widetilde{\xi}, h-\widetilde{h})\|+\psi(1)\|u(1)-\widetilde{u}(1)\| \\
& \left.+\int_{0}^{1}\left|\psi^{\prime}(t)\right|\|u(t)-\widetilde{u}(t)\| d t+\int_{0}^{1} \psi(t)\left\|f\left(t, u_{t}\right)-f\left(t, \widetilde{u}_{t}\right)\right\| d t\right] \\
& \leq \ell m^{-2}\|\psi\|_{L^{1}(0,1 ; \mathbb{R})}\left[\|u-\widetilde{u}\|_{C([0,1] ; X)}+\|h-\widetilde{h}\|_{C([-\tau, 0] ; X)}\right][\|\eta(\xi, h)\| \\
& \left.+\psi(1)\|u(1)\|+\int_{0}^{1}\left|\psi^{\prime}(t)\right|\|u(t)\| d t+\int_{0}^{1} \psi(t)\left\|f\left(t, u_{t}\right)\right\| d t\right] \\
& +m^{-1}\left\{\|\eta(\xi-\widetilde{\xi}, h-\widetilde{h})\|+\left[\psi(1)+\left\|\psi^{\prime}\right\|_{L^{1}(0,1 ; \mathbb{R})}\right]\|u-\widetilde{u}\|_{C([0,1] ; X)}\right. \\
& \left.+\ell_{1}\|\psi\|_{L^{1}(0,1 ; \mathbb{R})}\left[\|u-\widetilde{u}\|_{C([0,1] ; X)}+\|h-\widetilde{h}\|_{C([-\tau, 0] ; X)}\right]\right\} .
\end{aligned}
$$

We note that

$$
\|\eta(\xi-\widetilde{\xi}, h-\widetilde{h})\| \leq\|\widetilde{\xi}-\xi\|_{D(A)}+\|\psi\|_{C([0,1] ; \mathbb{R})}\|\widetilde{h}-h\|_{C([-\tau, 0] ; X)},
$$

for each $(\widetilde{\xi}, \widetilde{h}) \in D(A) \times C([-\tau, 0] ; X)$. 
5.2. The increments of the forcing term in (3.2)

Let us denote by $G$ the forcing term in (3.2), i.e.,

$$
G(u, \xi, h)(t)=\varphi\left(t, u_{t}\right) F(u, \xi, h)+f\left(t, u_{t}\right),
$$

for $t \in[0,1]$.

Let us recall that, in view of (2.14) and (2.9), we have

$$
\|F(u, \xi, h)\| \leq L_{0}\|u\|_{C([0,1] ; X)}+M_{0}(\xi, h),
$$

where $L_{0}$ and $M_{0}(\xi, h)$ are given by (2.15). We notice that $L_{0}$ is independent of both $\xi$ and $h$. Moreover, the mapping $(\xi, h) \mapsto M_{0}(\xi, h)$, defined as in (2.15), is continuous from $D(A) \times C([-\tau, 0] ; X), D(A)$ being endowed with the graph norm and $C([-\tau, 0] ; X)$ with the usual sup-norm. From the identity $G(u, \xi, h)(t)=\varphi\left(t, u_{t}\right) F(u, \xi, h)+f\left(t, u_{t}\right)-f(t, 0)+f(t, 0)$, for all $t \in[0,1]$, combined with (5.3), we deduce the following estimate (recall that, by $\left(f_{2}\right)$ in $\left(H_{f}\right)$, we have $\left.\|f(\cdot, 0)\|_{C([0,1] ; X)} \leq \ell_{2}\right)$ :

$$
\begin{aligned}
& \|G(u, \xi, h)(t)\| \leq m_{2}\left[L_{0}\|u\|_{C([0,1] ; X)}+M_{0}(\xi, h)\right]+\ell_{1}\|u\|_{C([0,1] ; X)} \\
& +\ell_{1}\|h\|_{C([-\tau, 0] ; X)}+\ell_{2} \\
& \leq\left(m_{2} L_{0}+\ell_{1}\right)\|u\|_{C([0,1] ; X)}+m_{2} M_{0}(\xi, h)+\ell_{1}\|h\|_{C([-\tau, 0] ; X)}+\ell_{2} .
\end{aligned}
$$

\section{blem $(\mathcal{J P})$}

\subsection{An a priori estimate for the mild solution of the pro-}

It is immediate to check out that $(\mathcal{J P})$ is equivalent to the following integral equation

$$
\begin{aligned}
u(t) & =S(t) h(0)+\int_{0}^{t} S(t-s) G(u, \xi, h)(s) d s \\
& =: S(t) h(0)+N(u, \xi, h)(t)
\end{aligned}
$$

for $t \in[0,1]$. Let us assume for the moment that:

$$
\|S(\cdot)\|_{L^{1}}<\left(m_{2} L_{0}+\ell_{1}\right)^{-1}
$$

where

$$
\|S(\cdot)\|_{L^{1}}:=\int_{0}^{1}\|S(s)\|_{\mathcal{L}(X)} d s .
$$


For a sufficient condition in order that (5.6) be satisfied see Remark 5.1 below. Then, from (5.4), we deduce

$$
\begin{aligned}
\|u(t)\| & \leq\|S(t) h(0)\|+\left(m_{2} L_{0}+\ell_{1}\right)\|u\|_{C([0,1] ; X)} \int_{0}^{t}\|S(s)\|_{\mathcal{L}(X)} d s \\
& +\left[m_{2} M_{0}(\xi, h)+\ell_{1}\|h\|_{C([-\tau, 0] ; X)}+\ell_{2}\right] \int_{0}^{t}\|S(s)\|_{\mathcal{L}(X)} d s
\end{aligned}
$$

for each $t \in[0,1]$. Whence, we get

$$
\begin{aligned}
& \|u\|_{C([0,1] ; X)} \leq\|S(\cdot) h(0)\|_{C([0,1] ; X)}+\left(m_{2} L_{0}+\ell_{1}\right)\|u\|_{C([0,1] ; X)}\|S(\cdot)\|_{L^{1}} \\
& +\left[m_{2} M_{0}(\xi, h)+\ell_{1}\|h\|_{C([-\tau, 0] ; X)}+\ell_{2}\right]\|S(\cdot)\|_{L^{1}} .
\end{aligned}
$$

This, in turn, implies

$$
\begin{aligned}
& \|u\|_{C([0,1] ; X)} \leq m_{3}^{-1}\|h(0)\|\|S(\cdot)\|_{C([0,1] ; \mathcal{L}(X))}+m_{3}^{-1}\left[m_{2} M_{0}(\xi, h)\right. \\
& \left.+\ell_{1}\|h\|_{C([-\tau, 0] ; X)}+\ell_{2}\right]\|S(\cdot)\|_{L^{1}}=: r_{1}(\xi, h),
\end{aligned}
$$

where

$$
m_{3}:=1-\left(m_{2} L_{0}+\ell_{1}\right)\|S(\cdot)\|_{L^{1}}
$$

and $\|S(\cdot)\|_{L^{1}}$ is defined in (5.7). Clearly, by (5.6), we deduce that $m_{3}>0$. In particular, we have shown that

$$
\begin{aligned}
& \|N(u, \xi, h)\|_{C([0,1] ; X)} \leq\left(m_{2} L_{0}+\ell_{1}\right)\|u\|_{C([0,1] ; X)}\|S(\cdot)\|_{L^{1}} \\
& +\left[m_{2} M_{0}(\xi, h)+\ell_{1}\|h\|_{C([-\tau, 0] ; X)}+\ell_{2}\right]\|S(\cdot)\|_{L^{1}} .
\end{aligned}
$$

Recalling the expression of $m_{3}$, we conclude that $r_{1}(\xi, h)$ is given by

$$
\begin{aligned}
r_{1}(\xi, h) & =\frac{\|h(0)\|\|S(\cdot)\|_{C([0,1] ; \mathcal{L}(X))}}{1-\left(m_{2} L_{0}+\ell_{1}\right)\|S(\cdot)\|_{L^{1}}} \\
& +\frac{\left[m_{2} M_{0}(\xi, h)+\ell_{1}\|h\|_{C([-\tau, 0] ; X)}+\ell_{2}\right]\|S(\cdot)\|_{L^{1}}}{1-\left(m_{2} L_{0}+\ell_{1}\right)\|S(\cdot)\|_{L^{1}}} .
\end{aligned}
$$

Consequently, from (5.3), (5.8) and (5.10), we get

$$
\|F(u, \xi, h)\|_{C([0,1] ; X)} \leq L_{0} r_{1}(\xi, h)+M_{0}(\xi, h)=: r_{2}(\xi, h) .
$$

Summing up, we have proved: 
Lemma 5.1. Under the condition (5.6) each solution of the equation (5.5) satisfies the estimate (5.9).

Remark 5.1. Assume that

$$
\|S(t)\|_{\mathcal{L}(X)} \leq e^{-\omega t},
$$

for each $t>0$ and some $\omega>0$ satisfying

$$
\omega \geq m_{2} L_{0}+\ell_{1}
$$

Then

$$
\|S(\cdot)\|_{L^{1}} \leq \omega^{-1}\left[1-e^{-\omega t}\right]<\omega^{-1} \leq\left(m_{2} L_{0}+\ell_{1}\right)^{-1}
$$

and thus (5.6) holds true.

Lemma 5.2. Under the condition (5.12) each solution of the equation (5.5) satisfies the estimate (5.9).

At this point, let us observe that, $u$ and $\widetilde{u}$ are the mild solutions of the following problems:

$$
\left\{\begin{array}{l}
u^{\prime}(t)=A u(t)+G(u, \xi, h)(t), \quad t \in(0,1), \\
u(0)=h(0)
\end{array}\right.
$$

and

$$
\left\{\begin{array}{l}
\widetilde{u}^{\prime}(t)=A \widetilde{u}(t)+G(\widetilde{u}, \widetilde{\xi}, \widetilde{h})(t), \quad t \in(0,1), \\
\widetilde{u}(0)=\widetilde{h}(0) .
\end{array}\right.
$$

5.4. A new estimate of $F(u, \xi, h)-F(\widetilde{u}, \widetilde{\xi}, \widetilde{h})$

From (5.2) and (5.9), we have

$$
\begin{aligned}
& \|F(u, \xi, h)-F(\widetilde{u}, \widetilde{\xi}, \widetilde{h})\| \\
& \leq \ell m^{-2}\|\psi\|_{L^{1}(0,1 ; \mathbb{R})}\left[\|u-\widetilde{u}\|_{C([0,1] ; X)}+\|h-\widetilde{h}\|_{C([-\tau, 0] ; X)}\right]\{\|\eta(\xi, h)\| \\
(5.14)+ & \left.\left.+\psi(1)+\left\|\psi^{\prime}\right\|_{L^{1}(0,1 ; \mathbb{R})}\right] r_{1}(\xi, h)+\|\psi\|_{L^{1}(0,1 ; \mathbb{R})} \ell_{2}\right\} \\
& +m^{-1}\left\{\|\eta(\xi-\widetilde{\xi}, h-\widetilde{h})\|+\left[\psi(1)+\left\|\psi^{\prime}\right\|_{L^{1}(0,1 ; \mathbb{R})}\right]\|u-\widetilde{u}\|_{C([0,1] ; X)}\right. \\
& \left.+\ell_{1}\|\psi\|_{L^{1}(0,1 ; \mathbb{R})}\left[\|u-\widetilde{u}\|_{C([0,1] ; X)}+\|h-\widetilde{h}\|_{C([-\tau, 0] ; X)}\right]\right\} \\
& =: r_{3}(\xi, h)\|u-\widetilde{u}\|_{C([0,1] ; X)}+r_{4}(\xi, h)\left[\|A \xi-A \widetilde{\xi}\|+\|h-\widetilde{h}\|_{C([-\tau, 0] ; X)}\right],
\end{aligned}
$$


where (recall that, by $\left(f_{2}\right)$ in $\left.\left(H_{f}\right),\|f(\cdot, 0,0)\|_{C([0,1] ; X)} \leq \ell_{2}\right)$

$$
\begin{aligned}
r_{3}(\xi, h)= & \ell m^{-2}\|\psi\|_{L^{1}(0,1 ; \mathbb{R})}\left\{\|A \xi\|+\psi(0)\|h\|_{C([-\tau, 0] ; X)}\right. \\
& \left.+\left[\psi(1)+\left\|\psi^{\prime}\right\|_{L^{1}(0,1 ; \mathbb{R})}\right] r_{1}(\xi, h)+\ell_{2}\|\psi\|_{L^{1}(0,1 ; \mathbb{R})}\right\} \\
& +m^{-1}\left[\psi(1)+\left\|\psi^{\prime}\right\|_{L^{1}(0,1 ; \mathbb{R})}+\ell_{1}\|\psi\|_{L^{1}(0,1 ; \mathbb{R})}\right] \\
r_{4}(\xi, h)= & \max \left\{\ell m ^ { - 2 } \| \psi \| _ { L ^ { 1 } ( 0 , 1 ; \mathbb { R } ) } \left[\|A \xi\|+\psi(0)\|h\|_{C([-\tau, 0] ; X)}\right.\right. \\
& \left.+\left[\psi(1)+\left\|\psi^{\prime}\right\|_{L^{1}(0,1 ; \mathbb{R})}\right] r_{1}(\xi, h)+\|\psi\|_{L^{1}(0,1 ; \mathbb{R})} \ell_{2}\right] \\
& \left.+m^{-1}\left[\max \{1, \psi(0)\}+\ell_{1}\|\psi\|_{L^{1}(0,1 ; \mathbb{R})}\right]\right\} .
\end{aligned}
$$

5.5. An estimate of $G(u, \xi, h)-G(\widetilde{u}, \widetilde{\xi}, \widetilde{h})$

Starting from the the identity

$$
\begin{aligned}
& G(u, \xi, h)(t)-G(\widetilde{u}, \widetilde{\xi}, \widetilde{h})(t)=\left[\varphi\left(t, u_{t}\right)-\varphi\left(t, \widetilde{u}_{t}\right)\right] F(u, \xi, h) \\
& +\varphi\left(t, \widetilde{u}_{t}\right)[F(u, \xi, h)-F(\widetilde{u}, \widetilde{\xi}, \widetilde{h})]+f\left(t, u_{t}\right)-f\left(t, \widetilde{u}_{t}\right),
\end{aligned}
$$

for each $t \in[0,1]$, from $\left(\varphi_{2}\right)$ in $\left(H_{\varphi}\right),(5.11),(5.14)$ and $(5.15)$, we deduce the following estimate:

$$
\begin{aligned}
& \|G(u, \xi, h)-G(\widetilde{u}, \widetilde{\xi}, \widetilde{h})\|_{C([0,1] ; X)} \\
& \leq\left[\ell r_{2}(\xi, h)+m_{2} r_{3}(\xi, h)+\ell_{1}\right]\|u-\widetilde{u}\|_{C([0,1] ; X)} \\
& +\left[m_{2} r_{4}(\xi, h)+\ell_{1}\right]\left[\|A \xi-A \widetilde{\xi}\|+\|h-\widetilde{h}\|_{C([-\tau, 0] ; X)}\right] .
\end{aligned}
$$

Let us observe that, for all $t \in[0,1]$,

$$
N(u, \xi, h)(t)-N(\widetilde{u}, \widetilde{\xi}, \widetilde{h})(t)=\int_{0}^{t} S(t-s)[G(u, \xi, h)(s)-G(\widetilde{u}, \widetilde{\xi}, \widetilde{h})(s)] d s
$$

So, for all $t \in[0,1]$, we have

$$
\begin{aligned}
& \|N(u, \xi, h)(t)-N(\widetilde{u}, \widetilde{\xi}, \widetilde{h})(t)\| \\
& \leq\left[\ell_{2}(\xi, h)+m_{2} r_{3}(\xi, h)+\ell_{1}\right]\|u-\widetilde{u}\|_{C([0,1] ; X)}\|S(\cdot)\|_{L^{1}} \\
& +\left[m_{2} r_{4}(\xi, h)+\ell_{1}\right]\left[\|A \xi-A \widetilde{\xi}\|+\|h-\widetilde{h}\|_{C([-\tau, 0] ; X)}\right]\|S(\cdot)\|_{L^{1}}
\end{aligned}
$$




\subsection{Uniqueness and continuous dependence on the data}

Let us denote by $C_{0, A}([-\tau, 0] ; X)$ the linear space of all functions $h \in$ $C([-\tau, 0] ; X)$ satisfying $h(0) \in D(A)$. Endowed with the norm

$$
\left.\|h\|_{0, A}=\sup \{\|h(t)\| ; t \in[-\tau, 0]\}+\|h(0)\|+\|A h(0)\|\right\}
$$

this is a Banach space.

As $r_{1}, \ldots r_{4}, M_{0}: D(A) \times C_{0, A}([-\tau, 0] ; X) \rightarrow(0,+\infty)$ are continuous whenever $D(A)$ and $C_{0, A}([-\tau, 0] ; X)$ are endowed, respectively, with the graph norm and the norm $\|\cdot\|_{0, A}$, from the estimates above, we deduce:

Theorem 5.1. In addition to the hypotheses of Theorem 3.1 assume that

$$
\|S(\cdot)\|_{L^{1}}<\min \left\{\left(m_{2} L_{0}+\ell_{1}\right)^{-1},\left[\ell r_{2}(\xi, h)+m_{2} r_{3}(\xi, h)+\ell_{1}\right]^{-1}\right\}
$$

and

$$
\omega>\max \left\{\left(m_{2} L_{0}+\ell_{1}\right),\left[\ell r_{2}(\xi, h)+m_{2} r_{3}(\xi, h)+\ell_{1}\right]\right\}
$$

are satisfied, where $r_{2}(\xi, h)$ is given by (5.11) and $r_{3}(\xi, h)$ is given by (5.15). Then, the mild solution $(z, u)$, whose existence is ensured by Theorem 3.1, is unique and the application $(\xi, h) \mapsto(z, u)$, from $D(A) \times C_{0, A}([-\tau, 0] ; X)$ to $X \times C([0,1] ; X)$, is locally Lipschitz. Here $D(A)$ is endowed with the graph norm, $C_{0, A}([-\tau, 0] ; X)$ with the norm $\|\cdot\|_{0, A}$ defined as above and $X \times C([0,1] ; X)$ with the norm $\|\cdot\|+\|\cdot\|_{C([0,1] ; X)}$.

Proof. If (5.17) and (5.18) are satisfied, then (5.13) holds true. So, all the estimates obtained before hold true.

Choose now $(\xi, h) \in D(A) \times C_{0, A}([-\tau, 0] ; X)$ satisfying (5.17) and (5.18). For a fixed $\omega$ satisfying (3.1) this is possible if $\omega$ satisfies (5.18) with $(\xi, h)=(0,0)$. Indeed,

$$
\left\{\begin{array}{l}
r_{1}(0,0)=\frac{\ell_{2} m_{2}\|\psi\|_{L^{1}(0,1 ; \mathbb{R})}\|S(\cdot)\|_{L^{1}}}{1-\left(m_{2} L_{0}+\ell_{1}\right)\|S(\cdot)\|_{L^{1}}}, \\
r_{2}(0,0)=L_{0} r_{1}(0,0)+\frac{\ell_{2}}{m}\|\psi\|_{L^{1}(0,1 ; \mathbb{R})}, \\
r_{3}(0,0)=\ell_{2} m^{-2}\|\psi\|_{L^{1}(0,1 ; \mathbb{R})}\left[|\psi(1)|+\left\|\psi^{\prime}\right\|_{L^{1}(0,1 ; \mathbb{R})}\right] r_{1}(0,0) \\
+\ell_{2}\|\psi\|_{L^{1}(0,1 ; \mathbb{R})}+m^{-1}\left[|\psi(1)|+\left\|\psi^{\prime}\right\|_{L^{1}(0,1 ; \mathbb{R})}+\ell_{1}\|\psi\|_{L^{1}(0,1 ; \mathbb{R})}\right] .
\end{array}\right.
$$

So, if $\xi$ and $h$ are small, $\omega$ satisfies (5.18). 
Then $u \mapsto N(u, \xi, h)$ is a contraction in the metric space

$$
K(\xi, h)=\left\{u \in C([0,1] ; X) ;\|u\|_{C([0,1] ; X)} \leq r_{1}(\xi, h)\right\} .
$$

Therefore, for any pair satisfying (5.17) and (5.18), the equation (5.5) admits a unique solution $u=U(\xi, h) \in C([0,1] ; X)$. Moreover, the operator $U$ depends continuously on the set of consisting of all pairs $(\xi, h)$ satisfying (5.17) and (5.18). Since $r_{1} \sim r_{4}$ depend continuously on $(\xi, h) \in$ $D(A) \times C_{0, A}([-\tau, 0] ; X)$ if $D(A)$ is endowed with the graph norm and on $C_{0, A}([-\tau, 0] ; X)$ with the norm $\|\cdot\|_{0, A}$, it follows that the set $\mathcal{D}$ of all pairs satisfying $(5.17)$ and $(5.18)$ is open. So, if $(\xi, h),(\widetilde{\xi}, \widetilde{h}) \in \mathcal{D}$, from (5.5) and (5.16), we deduce that

$$
\begin{aligned}
& \|U(\xi, h)-U(\widetilde{\xi}, \widetilde{h})\|_{C([0,1] ; X)} \\
& \leq\left\{\left[\ell_{2}(\xi, h)+m_{2} r_{3}(\xi, h)+\ell_{1}\right]\|U(\xi, h)(t)-U(\widetilde{\xi}, \widetilde{h})\|_{C([0,1] ; X)}\right. \\
& \left.+\left[m_{2} r_{4}(\xi, h)+\ell_{1}\right]\left[\|A \xi-A \widetilde{\xi}\|+\|h-\widetilde{h}\|_{C([-\tau, 0] ; X)}\right]\right\}\|S(\cdot)\|_{L^{1}},
\end{aligned}
$$

implying that

$$
\begin{aligned}
& \|U(\xi, h)-U(\widetilde{\xi}, \widetilde{h})\|_{C([0,1] ; X)} \leq\left[1-r_{5}(\xi, h)\right]^{-1}\left[m_{2} r_{4}(\xi, h)+\ell_{1}\right][\|A \xi-A \widetilde{\xi}\| \\
& +\|h-\widetilde{h}\|_{C([-\tau, 0] ; X)]}\|S(\cdot)\|_{L^{1}},
\end{aligned}
$$

where

$$
r_{5}(\xi, h)=\left[\ell r_{2}(\xi, h)+m_{2} r_{3}(\xi, h)+\ell_{1}\right]\|S(\cdot)\|_{L^{1}} .
$$

Whence $U$ is locally Lipschitz and this completes the proof.

\section{An application to an integrodifferential parabolic problem}

Let $\Omega$ in $\mathbb{R}^{n}$ be a bounded domain whose boundary $\partial \Omega$ is of class $C^{2}$. We will now apply the abstract results of the Sections 3 and 6 to recover the unknown function $u:[0, T] \times \Omega \rightarrow \mathbb{R}$ and the coefficient $z: \Omega \rightarrow \mathbb{R}$ in the following integrodifferential parabolic identification problem:

$\left(\mathcal{J P}_{1}\right)$ Find $z \in L^{q}(\Omega)$ and $u:[-\tau, 1] \rightarrow L^{q}(\Omega)$ satisfying

$$
\left\{\begin{array}{l}
\frac{\partial u}{\partial t}(t, x)=\mathcal{A} u(t, x)+z(x) \varphi\left(t, u_{t}\right) \\
+f\left(t, u_{t}\right)(x), \quad(t, x) \in[0,1] \times \Omega, \\
B u(t, x)=0, \quad(t, x) \in[0,1] \times \partial \Omega, \\
u(t, x)=h(t, x), \quad(t, x) \in(-\tau, 0] \times \Omega,
\end{array}\right.
$$


and the additional condition

$$
\int_{0}^{1} \psi(t) u(t, x) d t=\xi(x), \quad x \in \Omega,
$$

where the functional $\varphi$ and the operator $f$ are as in the two cases described below.

Case 1. The functional $\varphi$ is of the form

$$
\varphi(t, v)=\sigma\left(t, \int_{(-\tau, 0) \times \Omega} v(s, y) d s d y\right),
$$

for each $t \in[0,1]$ and each $v \in C\left([-\tau, 0] ; L^{q}(\Omega)\right)$, and

$$
\begin{aligned}
f(t, v)(x) & =g_{0}\left(t, x, \int_{-\tau}^{0} v(s, x) d s\right)+\int_{\Omega} g_{1}\left(t, x, y, \int_{-\tau}^{0} v(s, y) d s\right) d y \\
& =f_{0}(t, v)(x)+f_{1}(t, v)(x)
\end{aligned}
$$

for each $t \in[0,1]$, each $v \in C\left([-\tau, 0] ; L^{q}(\Omega)\right)$ and for a.e. $x \in \Omega$, where $\sigma:[0,1] \times \mathbb{R} \rightarrow \mathbb{R}, g_{0}:[0,1] \times \Omega \times \mathbb{R} \rightarrow \mathbb{R}$ and $g_{1}:[0,1] \times \Omega \times \Omega \times \mathbb{R} \rightarrow \mathbb{R}$ are given functions satisfying the properties:

$\left(H^{\sigma}\right) \sigma \in C^{0,1}([0,1] \times \mathbb{R})^{2}$ and there exist two positive constants $m_{1}, m_{2}$ such that $m_{1} \leq \sigma(t, v) \leq m_{2}$ for all $(t, v) \in[0,1] \times \mathbb{R}$;

$\left(H^{g_{0}}\right)$ for each $(t, v) \in[0,1] \times \mathbb{R}$, the function $x \mapsto g_{0}(t, x, v)$ is measurable and there exists $l_{0}$ such that

$$
\left|g_{0}(t, x, v)-g_{0}(\tilde{t}, x, \tilde{v})\right| \leq l_{0}[|t-\tilde{t}|+|v-\tilde{v}|]
$$

for all $t, \tilde{t} \in[0,1], v, \tilde{v} \in \bar{\Omega}$ and a.e. for $x \in \Omega$;

$\left(H^{g_{1}}\right)$ for each $(t, v) \in[0,1] \times \mathbb{R}$, the function $(x, y) \mapsto g_{1}(t, x, y, v)$ is measurable and there exists $l_{1}: \Omega \times \Omega \rightarrow \mathbb{R}$ such that, a.e. for $y \in \Omega, x \mapsto l_{1}(x, y)$ belongs to $L^{q}(\Omega), y \mapsto\left\|l_{1}(\cdot, y)\right\|_{L^{q}(\Omega)}$ belongs to $L^{q^{\prime}}(\Omega)$ and

$$
\left|g_{1}(t, x, y, v)-g_{1}(\tilde{t}, x, y, \tilde{v})\right| \leq l_{1}(x, y)[|t-\tilde{t}|+|v-\tilde{v}|]
$$

for all $t, \tilde{t} \in[0,1], v, \tilde{v} \in \bar{\Omega}$ and for a.e. $x \in \Omega$ and $y \in \Omega$.

\footnotetext{
${ }^{2} C^{0,1}([0,1] \times \mathbb{R})$ denotes the Banach space of all Lipschitz-continuous functions in $[0,1] \times \mathbb{R}$.
} 
Case 2. The functional $\varphi$ is of the form

$$
\varphi(t, v)=\int_{(-\tau, 0) \times \Omega} \sigma(t, s, y, v(s, y)) d s d y,
$$

for each $t \in[0,1]$ and each $v \in C\left([-\tau, 0] ; L^{q}(\Omega)\right)$, and

$$
\begin{aligned}
f(t, v)(x) & =\int_{-\tau}^{0} g_{0}(t, s, x, v(s, x)) d s+\int_{(-\tau, 0) \times \Omega} g_{1}(t, s, x, y, v(s, y)) d s d y \\
& =f_{0}(t, v)(x)+f_{1}(t, v)(x)
\end{aligned}
$$

for each $t \in[0,1]$, each $v \in C\left([-\tau, 0] ; L^{q}(\Omega)\right)$ and a.e. for $x \in \Omega$, where $\sigma:[0,1] \times[-\tau, 0] \times \Omega \times \mathbb{R} \rightarrow \mathbb{R}, g_{0}:[0,1] \times[-\tau, 0] \times \Omega \times \mathbb{R} \rightarrow \mathbb{R}$ and $g_{1}:[0,1] \times[-\tau, 0] \times \Omega \times \Omega \times \mathbb{R} \rightarrow \mathbb{R}$ are given functions satisfying the properties:

$\left(H^{\sigma, 1}\right)$ for all $(t, v) \in[0,1] \times \mathbb{R}$, the function $(s, y) \mapsto \sigma(t, s, y, v)$ is measurable;

$\left(H^{\sigma, 2}\right)$ there exist $\sigma_{1}, \sigma_{2} \in L^{1}((-\tau, 0) \times \Omega) \backslash\{0\}$ such that

$$
0 \leq \sigma_{1}(s, y) \leq \sigma(t, s, y, v) \leq \sigma_{2}(s, y)
$$

for all $(t, v) \in[0,1] \times \mathbb{R}$ and for a.e. $(s, y) \in(-\tau, 0) \times \Omega$;

$\left(H^{\sigma, 3}\right)$ there exists a nonnegative function $l \in L^{q^{\prime}}((0,1) \times \Omega)$ such that

$$
|\sigma(t, s, y, v)-\sigma(\tilde{t}, s, y, \tilde{v})| \leq l(s, y)[|t-\tilde{t}|+|v-\tilde{v}|]
$$

for all $t, \tilde{t} \in[0,1], v, \tilde{v} \in \bar{\Omega}$ and for a.e. $(s, y) \in(0,1) \times \Omega$;

$\left(H^{g_{0}, 1}\right)$ for all $(t, v) \in[0,1] \times \mathbb{R}$, the function $(s, y) \mapsto g_{0}(t, s, x, v)$ is measurable;

$\left(H^{g_{0}, 2}\right)$ there exists a function $l_{0} \in L^{1}(0,1)$ such that

$$
\left|g_{0}(t, s, x, v)-g_{0}(\tilde{t}, s, x, \tilde{v})\right| \leq l_{0}(s)[|t-\tilde{t}|+|v-\tilde{v}|]
$$

for all $t, \tilde{t} \in[0,1], v, \tilde{v} \in \bar{\Omega}$ and for a.e. $(s, x) \in(0,1) \times \Omega$;

$\left(H^{g_{0}, 3}\right)$ the function $t \mapsto \int_{-\tau}^{0}\left\|g_{0}(t, s, \cdot, 0)\right\|_{L^{q}(\Omega)} d s$ is bounded on $(0,1) \times \Omega$; 
$\left(H^{g_{1}, 1}\right)$ for all $(t, v) \in[0,1] \times \mathbb{R}$, the function $(s, x, y) \mapsto g_{1}(t, s, x, y, v)$ is measurable;

$\left(H^{g_{1}, 2}\right)$ there exists a nonnegative measurable function $l_{1}:[-\tau, 0] \times \Omega \times \Omega$ satisfying $\int_{-\tau}^{0} d s\left[\int_{\Omega}\left\|l_{1}(s, \cdot, y)\right\|_{L^{q}(\Omega)}^{q^{\prime}} d y\right]^{1 / q^{\prime}} d s<+\infty$ and such that

$$
\left|g_{1}(t, s, x, y, v)-g_{1}(\tilde{t}, s, x, y, \tilde{v})\right| \leq l_{1}(s, x, y)[|t-\tilde{t}|+|v-\tilde{v}|]
$$

for all $t, \tilde{t} \in[0,1], v, \tilde{v} \in \bar{\Omega}$ and for a.e. $(s, x) \in[-\tau, 0] \times \Omega$;

$\left(H^{g_{1}, 3}\right)$ the function

$$
t \mapsto \int_{(-\tau, 0) \times \Omega}\left\|g_{1}(t, s, \cdot, y, 0)\right\|_{L^{q}(\Omega)} d s d y
$$

is bounded on $[0,1]$.

Of course, one could also consider some combinations of the two cases above, i.e. that some terms are as in Case $\mathbf{1}$ and some as in Case 2. However, for the sake of simplicity, we limit ourselves to dealing with Cases 1 and 2, only.

Now we observe that in problem $(6.1) \mathcal{A}$ denotes the following secondorder linear symmetric differential operator in $\Omega$

$$
\mathcal{A}=\sum_{j, k=1}^{n} D_{x_{j}}\left(a_{j, k}(x) D_{x_{k}}\right)-a_{0}(x)
$$

We assume that $a_{0} \in C(\bar{\Omega})$ and $a_{j, k} \in C^{1}(\bar{\Omega}), a_{i, j}=a_{j, i}, j, k=1, \ldots, n$, satisfy

$$
a_{0}(x) \geq \omega>0, \quad \forall x \in \bar{\Omega}, \quad \sum_{j, k=1}^{n} a_{j, k}(x) \xi_{j} \xi_{k} \geq \mu|\xi|^{2}, \quad \forall(x, \xi) \in \bar{\Omega} \times \mathbb{R}^{n},
$$

$\mu$ being a positive constant.

Moreover, the operator $B$ is defined by either of the following relations:

$$
\begin{aligned}
& \text { (D) } B w(x)=w(x), \quad x \in \partial \Omega, \\
& \text { (R) } B w(x)=D_{\nu_{\mathcal{A}}} w(x)+b_{0}(x) w(x), \quad x \in \partial \Omega,
\end{aligned}
$$


where $b_{0} \in C(\bar{\Omega}), b_{0}(x) \geq 0$ for all $x \in \partial \Omega$, and $\nu_{\mathcal{A}}$ is the conormal vector associated with $\mathcal{A}$, i.e. $\left(\nu_{\mathcal{A}}(x)\right)_{j}=\sum_{j, k=1}^{n} \nu_{i}(x) a_{j, k}(x), \nu(x)$ denoting the outward normal vector at $x \in \partial \Omega$.

Here (D) and (R) stand, respectively, for the Dirichlet and the Robin boundary operators.

Then, related to the reference space $X_{q}=L^{q}(\Omega), q \in(1,+\infty)$, we define

$$
\begin{aligned}
& \left.\mathcal{D}\left(A_{q}\right)=\left\{w \in W^{2, q}(\Omega)\right): B w=0 \text { on } \partial \Omega\right\}, \\
& A_{q} w=\mathcal{A} w, \quad w \in \mathcal{D}\left(A_{q}\right),
\end{aligned}
$$

the operator $B$ being defined by (6.3) and (6.4).

Then it is well-known (cf. e.g. AnIKonov and Lorenzi [1]) that $A_{q}$ generates in $L^{q}(\Omega)$ the semigroup $\left\{e^{A_{q} t}\right\}_{t \geq 0}$, which is analytic in the sector $\Sigma_{(\pi / 2)-\omega_{p}}$ and satisfies the estimate

$$
\left\|e^{A_{q} t}\right\|_{\mathcal{L}(X)} \leq e^{-\rho_{0} t}, \quad t \in \Sigma_{(\pi / 2)-\omega_{q}} .
$$

Moreover, from VRABIE [13, Proposition 2.2.1, p. 41 $]^{3}$, taking into account that $\|u\|:=\|u\|_{L^{q}(\Omega)}+\|\mathcal{A} u\|_{L^{q}(\Omega)}$ is a norm on $W^{2, q}(\Omega)$ ) equivalent to the usual one, and using the Sobolev, Rellich-Kondrachov Theorem - see VRABIE [13, Theorem 1.3.8, p. 10], we deduce that $(I-A)^{-1}$ is a compact operator from $L^{q}(\Omega)$ into itself.

Theorem 6.1. Let the assumptions listed in Cases 1 and 2 be satisfied and let $\omega$ satisfy inequality (5.18). Then, the problem $\left(\mathcal{J P}_{1}\right)$ has a unique mild solution $(u, z) \in\left[C^{1}\left((0,1) ; L^{q}(\Omega)\right) \cap C([0, T] ; \mathcal{D}(A))\right] \times L^{q}(\Omega), q \in$ $(1,+\infty)$, continuously depending on the data.

Proof. We consider separately Case 1 and Case 2. We recall that, in both cases, $X=L^{q}(\Omega)$.

Case 1. The property $\left(\varphi_{1}\right)$ in $\left(H^{\varphi}\right)$ immediately follows from the inequality in $\left(H^{\sigma}\right)$, while property $\left(\varphi_{2}\right)$ is implied by the inequalities

$$
\begin{aligned}
|\varphi(t, v)-\varphi(\tilde{t}, \tilde{v})| & \leq\left|\sigma\left(t, \int_{(-\tau, 0) \times \Omega} v(s, y) d s d y\right)-\sigma\left(\tilde{t}, \int_{(-\tau, 0) \times \Omega} \tilde{v}(s, y) d s d y\right)\right| \\
& \leq l\left[|t-\tilde{t}|+\int_{(-\tau, 0) \times \Omega}|(v-\tilde{v})(s, y)| d s d y\right] \\
& \leq l\left[|t-\tilde{t}|+\tau m(\Omega)^{1 / q^{\prime}}\|v-\tilde{v}\|_{C\left([0,1] ; L^{q}(\Omega)\right)}\right],
\end{aligned}
$$

\footnotetext{
${ }^{3}$ According to Proposition 2.2.1, $(I-A)^{-1}$ is compact if and only if for each $k>0$,
} the level set $\ell_{k}=\{u \in D(A) ;\|u\|+\|A u\| \leq k\}$ is relatively compact in $X$. 
$l$ being the Lipschitz constant related to $\sigma$.

So, we have proved our assertion with

$$
\ell=l \max \left\{1, \tau m(\Omega)^{1 / q^{\prime}}\right\} .
$$

Then, from the inequality

$$
\begin{aligned}
\left|f_{0}(t, v)(x)-f_{0}(\tilde{t}, \tilde{v})(x)\right| & =\left|g_{0}\left(t, x, \int_{-\tau}^{0} v(s, x) d s\right)-g_{0}\left(\tilde{t}, x, \int_{-\tau}^{0} \tilde{v}(s, y) d s\right)\right| \\
& \leq l_{0}\left[|t-\tilde{t}|+\int_{(-\tau, 0) \times \Omega}|(v-\tilde{v})(s, x)| d s\right],
\end{aligned}
$$

we get

$$
\left\|f_{0}(t, v)-f_{0}(\tilde{t}, \tilde{v})\right\|_{\left.L^{q}(\Omega)\right)} \leq l_{0}\left[m(\Omega)^{1 / q}|t-\tilde{t}|+\tau\|v-\tilde{v}\|_{C\left([0,1] ; L^{q}(\Omega)\right)}\right] .
$$

Similarly, from the inequality

$$
\begin{aligned}
& \left|f_{1}(t, v)(x)-f_{1}(\tilde{t}, \tilde{v})(x)\right| \\
& \leq \int_{\Omega}\left|g_{1}\left(t, x, y, \int_{-\tau}^{0} v(s, y) d s\right)-g_{1}\left(\tilde{t}, x, y, \int_{-\tau}^{0} \tilde{v}(s, y) d s\right)\right| d y \\
& \leq \int_{\Omega} l_{1}(x, y)\left[|t-\tilde{t}|+\int_{-\tau}^{0}|(v-\tilde{v})(s, y)| d s\right] d y,
\end{aligned}
$$

we obtain

$$
\begin{aligned}
& \left\|f_{1}(t, v)-f_{1}(\tilde{t}, \tilde{v})\right\|_{L^{q}(\Omega)} \\
& \leq|t-\tilde{t}| \int_{\Omega}\left\|l_{1}(\cdot, y)\right\|_{L^{q}(\Omega)}+\int_{-\tau}^{0} d s \int_{\Omega}\left\|l_{1}(\cdot, y)\right\|_{L^{q}(\Omega)}|(v-\tilde{v})(s, y)| d y \\
& \leq|t-\tilde{t}| \int_{\Omega}\left\|l_{1}(\cdot, y)\right\|_{L^{q}(\Omega)} d y \\
& +\|v-\tilde{v}\|_{C\left([0,1] ; L^{q}(\Omega)\right)} \int_{-\tau}^{0}\left[\int_{\Omega}\left\|l_{1}(\cdot, y)\right\|_{L^{q}(\Omega)}^{q^{\prime}} d y\right]^{1 / q^{\prime}} d s .
\end{aligned}
$$

From the previous estimate we easily deduce the property $\left(f_{1}\right)$ in $\left(H_{f}\right)$ with

$$
\begin{aligned}
\ell_{1} & =\max \left\{l_{0} m(\Omega)^{1 / q}+\int_{\Omega}\left\|l_{1}(\cdot, y)\right\|_{L^{q}(\Omega)} d y, \tau\right. \\
& \left.+\int_{-\tau}^{0}\left[\int_{\Omega}\left\|l_{1}(\cdot, y)\right\|_{L^{q}(\Omega)}^{q^{\prime}} d y\right]^{1 / q^{\prime}} d s\right\} .
\end{aligned}
$$


Finally, from the definition of $\varphi$, we easily obtain the property $\left(f_{2}\right)$ with

$$
\ell_{2}=\max \left\{\left\|g_{0}(\cdot, \cdot, 0)\right\|_{C\left([0,1] ; L^{q}(\Omega)\right)}, \sup _{t \in[0,1]} \int_{\Omega}\left\|g_{1}(t, \cdot, y, 0)\right\|_{L^{q}(\Omega)} d y\right\} .
$$

Whence, we have checked that $\left(H_{\varphi}\right)$ and $\left(H_{f}\right)$ are fulfilled.

Case 2. We have to show that $\left(H_{\varphi}\right)$ and $\left(H_{f}\right)$ are fulfilled.

According to $\left(H^{\sigma_{2}}\right)$, the sub-condition $\left(\varphi_{2}\right)$ in $\left(H_{\varphi}\right)$ is satisfied with

$$
m_{j}=\int_{(-\tau, 0) \times \Omega} \sigma_{j}(y) d y, \mathrm{~J}=1,2,
$$

while the sub-condition $\left(\varphi_{1}\right)$ is implied from the following inequalities:

$$
\begin{aligned}
& |\varphi(t, v)-\varphi(\tilde{t}, \tilde{v})| \leq \int_{(-\tau, 0) \times \Omega}|\sigma(t, s, y, v(s, y))-\sigma(\tilde{t}, s, y, \tilde{v}(s, y))| d s d y \\
& \leq \int_{(-\tau, 0) \times \Omega} l(s, y)[|t-\tilde{t}|+|(v-\tilde{v})(s, y)|] d s d y \\
& \leq|t-\tilde{t}|\|l\|_{\left.(-\tau, 0) \times L^{1}(\Omega)\right)}+\int_{-\tau}^{0}\|l(s, \cdot)\|_{L^{q^{\prime}}(\Omega)}\|(v-\tilde{v})(s, \cdot)\|_{L^{q}(\Omega)} d s \\
& \leq|t-\tilde{t}|\|l\|_{\left.(-\tau, 0) \times L^{1}(\Omega)\right)}+\|v-\tilde{v}\|_{C\left([-\tau, 0] ; L^{q}(\Omega)\right)} \int_{-\tau}^{0}\|l(s, \cdot)\|_{L^{q^{\prime}}(\Omega)} d s .
\end{aligned}
$$

In this case we have

$$
\ell=\max \left\{\|l\|_{(-\tau, 0) \times L^{1}(\Omega)}, \int_{-\tau}^{0}\|l(s, \cdot)\|_{L^{q^{\prime}}(\Omega)} d s\right\} .
$$

Likewise, from the inequality

$$
\begin{aligned}
\left|f_{0}(t, v)(x)-f_{0}(\tilde{t}, \tilde{v})(x)\right| & \leq \int_{-\tau}^{0}\left|g_{0}(t, s, x, v(s, x))-g_{0}(\tilde{t}, s, x, \tilde{v}(s, x))\right| d s \\
& \leq \int_{-\tau}^{0} l_{0}(s)[|t-\tilde{t}|+|(v-\tilde{v})(s, x)|] d s
\end{aligned}
$$

we deduce

$$
\begin{aligned}
& \left\|f_{0}(t, v)-f_{0}(\tilde{t}, \tilde{v})\right\|_{L^{q^{\prime}}(\Omega)} \\
& \leq|t-\tilde{t}|\left\|l_{0}\right\|_{L^{1}(-\tau, 0)} m(\Omega)^{1 / q}+\int_{-\tau}^{0} l_{0}(s)\|(v-\tilde{v})(s, \cdot)\|_{L^{q}(\Omega)} d s \\
& \leq|t-\tilde{t}|\left\|l_{0}\right\|_{L^{1}(-\tau, 0)} m(\Omega)^{1 / q}+\left\|l_{0}\right\|_{L^{1}(-\tau, 0)}\|v-\tilde{v}\|_{C\left([-\tau, 0] ; L^{q}(\Omega)\right)} .
\end{aligned}
$$


Similarly, from the inequality

$$
\begin{aligned}
& \left|f_{1}(t, v)(x)-f_{1}(\tilde{t}, \tilde{v})(x)\right| \\
& \leq \int_{(-\tau, 0) \times \Omega}\left|g_{1}(t, x, y, v(s, y))-g_{1}(\tilde{t}, x, y, \tilde{v}(s, y))\right| d s d y \\
& \leq \int_{(-\tau, 0) \times \Omega} l_{1}(s, x, y)[|t-\tilde{t}|+|(v-\tilde{v})(s, y)|] d s d y,
\end{aligned}
$$

we get

$$
\begin{aligned}
& \left\|f_{1}(t, v)-f_{1}(\tilde{t}, \tilde{v})\right\|_{L^{q}(\Omega)} \\
& \leq|t-\tilde{t}| \int_{(-\tau, 0) \times \Omega}\left\|l_{1}(s, \cdot, y)\right\|_{L^{q}(\Omega)} d s d y \\
& +\int_{-\tau}^{0} d s \int_{\Omega}\left\|l_{1}(s, \cdot, y)\right\|_{L^{q}(\Omega)}|(v-\tilde{v})(s, y)| d s d y \\
& \leq|t-\tilde{t}| \int_{\Omega}\left\|l_{1}(s, \cdot, y)\right\|_{L^{q}(\Omega)} d s d y \\
& \left.+\int_{-\tau}^{0}\left[\int_{\Omega}\left\|l_{1}(s, \cdot, y)\right\|_{L^{q}(\Omega)}^{q^{\prime}} d y\right]^{1 / q^{\prime}} \| v-\tilde{v}\right)(s, \cdot) \|_{L^{q}(\Omega)} d s \\
& \leq|t-\tilde{t}| \int_{\Omega}\left\|l_{1}(s, \cdot, y)\right\|_{L^{q}(\Omega)} d s d y \\
& +\|v-\tilde{v}\|_{C\left([0,1] ; L^{q}(\Omega)\right)} \int_{-\tau}^{0}\left[\int_{\Omega}\left\|l_{1}(s, \cdot, y)\right\|_{L^{q}(\Omega)}^{q^{\prime}} d y\right]^{1 / q^{\prime}} d s .
\end{aligned}
$$

Summing up, we have shown that the sub-condition $\left(f_{1}\right)$ in $\left(H_{f}\right)$ holds true with

$$
\begin{aligned}
\ell_{1}=\max \left\{m(\Omega)^{1 / q}\left\|l_{0}\right\|_{L^{1}(-\tau, 0)}+\int_{\Omega}\left\|l_{1}(s, \cdot, y)\right\|_{L^{q}(\Omega)} d s d y,\right. \\
\left.\left\|l_{0}\right\|_{L^{1}(-\tau, 0)}+\int_{-\tau}^{0}\left[\int_{\Omega}\left\|l_{1}(s, \cdot, y)\right\|_{L^{q}(\Omega)}^{q^{\prime}} d y\right]^{1 / q^{\prime}} d s\right\} .
\end{aligned}
$$

Finally, according to $\left(H^{g_{0}, 3}\right)$ and $\left(H^{g_{1}, 4}\right)$, the sub-condition $\left(f_{2}\right)$ in $\left(H_{f}\right)$ holds with any

$$
\begin{aligned}
\ell_{2} & =\sup _{t \in[0,1]}\left\|g_{0}(t, \cdot, \cdot, 0)\right\|_{L^{1}\left((-\tau, 0) ; L^{q}(\Omega)\right)} \\
& +\sup _{t \in[0,1]} \int_{\Omega}\left\|g_{1}(t, \cdot, \cdot, y, 0)\right\|_{L^{1}\left((-\tau, 0) ; L^{q}(\Omega)\right)} d y
\end{aligned}
$$


as it easily follows from the formula

$$
f(t, v)(x)=\int_{-\tau}^{0} g_{0}(t, s, x, 0) d s+\int_{(-\tau, 0) \times \Omega} g_{1}(t, s, x, y, 0) d s d y .
$$

Then, by virtue of Theorem 5.1 and we can conclude that the problem (6.1), (6.2) admits a unique mild solution $(u, z)$ continuously depending on the data with respect to the norms pointed out in the statement of Theorem 5.1. The proof is complete.

\section{Application to a semilinear hyperbolic problem}

Finally, we give a simple example of an identification problem in which the operator $A$ does not generate a compact semigroup but, nevertheless, $(I-A)^{-1}$ is a compact operator. Let $C_{\pi}^{k}, k=1,2$, be the space of all $\pi$-periodic functions $u \in C^{k}(\mathbb{R} ; \mathbb{R})$.

Let $a \in \mathbb{R} \backslash\{0\}, \omega>0, \varphi:[0,1] \times C\left([-\tau, 0] ; C_{\pi}^{0}\right) \rightarrow \mathbb{R}_{+}$be a Lipschitz functional, $f:[0,1] \times C\left([-\tau, 0] ; C_{\pi}^{0}\right) \rightarrow C_{\pi}^{0}$ be a Lipschitz function, $\psi \in$ $W^{1,1}([0,1] ; \mathbb{R})$, and let us consider the following identification problem for the transport functional equation with delay, in $C_{\pi}^{0}$ :

$\left(\mathcal{J P}_{2}\right)$ Let $a \in \mathbb{R} \backslash\{0\}$ and $\omega>0$. Given $h \in C\left([-\tau, 0] ; C_{\pi}^{0}\right), \xi_{0}, \in C_{\pi}^{0}$ and $\psi \in\left[W^{1,1}([0,1] ; \mathbb{R}) \backslash\{0\}\right]$, find $z \in C_{\pi}^{0}$ and a mild solution $u:[0,1] \rightarrow C_{\pi}^{0}$ of the Cauchy Problem

$$
\left\{\begin{aligned}
\frac{\partial u}{\partial t}(t, x)= & \left.-a \frac{\partial u}{\partial x}(t, x)-\omega u(t, x)+\varphi\left(t, u_{t}(\cdot, x)\right)\right) z(x) \\
& +f\left(t, u_{t}(\cdot, x)\right), \quad(t, x) \in(0,1) \times \mathbb{R}^{n} \\
u(t, x)= & u(t, x+\pi), \quad(t, x) \in(0,1) \times \mathbb{R}^{n} \\
u(t, x)= & h(t, x), \quad t \in[-\tau, 0], \quad x \in(0, \pi)
\end{aligned}\right.
$$

satisfying the additional condition

$$
\int_{0}^{1} \psi(t) u(t, x) d t=\xi(x)
$$

Throughout, we denote by $\|\cdot\|_{\infty}$ the usual sup-norm on $C_{\pi}^{0}$.

From Theorem 3.1, we deduce

Theorem 7.1. Let $\omega>0, \varphi:[0,1] \times C\left([-\tau, 0] ; C_{\pi}^{0}\right) \rightarrow \mathbb{R}_{+}$be a $C^{1}$-functional, $f:[0,1] \times C\left([-\tau, 0] ; C_{\pi}^{0}\right) \rightarrow C_{\pi}^{0}$ be a $C^{1}$-function and $\psi \in$ 
$\left[W^{1,1}([0,1] ; \mathbb{R}) \backslash\{0\}\right]$ satisfying $\psi(t) \geq 0$ for each $t \in[0,1]$. Let us assume that there exist $\ell>0, \ell_{1}>0, \ell_{2}>0, m_{1}>0$ and $m_{2}>0$ such that

$$
\begin{aligned}
& |\varphi(t, v)-\varphi(\widetilde{t}, \widetilde{v})| \leq \ell\left[|t-\widetilde{t}|+\|v-\widetilde{v}\|_{C\left([-\tau, 0] ; C_{\pi}^{0}\right)}\right], \\
& m_{1} \leq \varphi(t, v) \leq m_{2}, \\
& \|f(t, v)-f(\widetilde{t}, \widetilde{v})\|_{\infty} \leq \ell_{1}\left[|t-\widetilde{t}|+\|v-\widetilde{v}\|_{C\left([-\tau, 0] ; C_{\pi}^{0}\right)}\right]
\end{aligned}
$$

for each $(t, v),(\widetilde{t}, \widetilde{v}) \in[0,1] \times C\left([-\tau, 0] ; C_{\pi}^{0}\right)$ and

$$
\|f(t, 0)\|_{\infty} \leq \ell_{2}
$$

for each $t \in[0,1]$. Let $h \in C\left([-\tau, 0] ; C_{\pi}^{0}\right)$ with $h(0) \in C_{\pi}^{1}$ and let us assume that (3.1) holds true. Then, there exists a mild solution $(z, u)$ to the problem $\left(\mathrm{JP}_{2}\right)$, admitting the implicit representation given by

$$
u(t, x)=h(t, x)
$$

for $t \in[-\tau, 0]$ and $x \in \mathbb{R}$,

$$
\begin{aligned}
u(t, x) & =e^{-\omega t} h(0, x-a t)+\int_{0}^{t} e^{-\omega(t-s)} f\left(s, u_{s}(\cdot, x-a(t-s))\right) d s \\
& +\int_{0}^{t} e^{-\omega(t-s)} \varphi\left(u\left(s, u_{s}(\cdot, x-a(t-s))\right) z_{s}(x-a(t-s)) d s,\right.
\end{aligned}
$$

where

$$
z=F(u)
$$

$F$ being defined by (2.5) and (2.6).

Let us assume further that $\omega>\ell_{1}$ is sufficiently large as to satisfy

$$
\frac{1-e^{-\omega}}{\omega}<\min \left\{\left(m_{2} L_{0}+\ell_{1}\right)^{-1},\left[\ell r_{2}(\xi, h)+m_{2} r_{3}(\xi, h)+\ell_{1}\right]^{-1}\right\}
$$

and

$$
\omega>\max \left\{\left(m_{2} L_{0}+\ell_{1}\right),\left[\ell r_{2}(\xi, h)+m_{2} r_{3}(\xi, h)+\ell_{1}\right]\right\},
$$

where $r_{2}(\xi, h)$ is given by (5.11) and $r_{3}(\xi, h)$ is given by (5.15). Then, the mild solution above is unique and the application $(\xi, h) \mapsto(z, u)$ from $C_{\pi}^{1} \times C_{0, A}\left([0,1] ; C_{\pi}^{0}\right)$ to $C_{\pi}^{0} \times C\left([0,1] ; C_{\pi}^{0}\right)$ is locally Lipschitz. Here $A$ : $C_{\pi}^{1} \subseteq C_{\pi}^{0} \rightarrow C_{\pi}^{0}$ is defined by $A u=-a u^{\prime}-\omega u$, for each $u \in C_{\pi}^{1}$. 
Proof. Let us observe that $\left(\mathcal{J P}_{3}\right)$ can be equivalently rewritten as $\left(\mathcal{J P}_{1}\right)$ in $X=C_{\pi}^{0}$, where $A, \varphi, f, \psi$ are as above. Clearly $A=A_{1}-\omega I$, where $A_{1} u=-a u^{\prime}$ for each $u \in C_{\pi}^{1}$. By VRABIE [14, Problem 3.4, p. 74], we know that $A_{1}$ generates a $C_{0}$-group of isometries on $C_{\pi}^{0},\{T(t) ; t \in \mathbb{R}\}$, defined by

$$
[T(t) \xi](x)=\xi(x-a t)
$$

for each $\xi \in C_{\pi}^{0}$, each $x \in \mathbb{R}$ and each $t \in \mathbb{R}$. Thus $A$ generates a $C_{0^{-}}$ semigroup of contractions on $C_{\pi}^{0},\{S(t) ; t \geq 0\}$. One may easily verify that

$$
S(t)=e^{-\omega t} T(t)
$$

for each $t \geq 0$. Moreover, by the infinite dimensional version of ArzelàAscoli's Theorem - see VRABIE [13, Theorem 1.3.1, p. 7] - it follows that for each $k>0$, the set

$$
\left\{u \in D(A) ;\|u\|_{\infty}+\|A u\|_{\infty} \leq k\right\}
$$

is relatively compact in $C_{\pi}^{0}$. By VRABIE [13, Proposition 2.2.1, p. 41], we deduce that $(I-A)^{-1}$ is compact. So, from this remark and $(7.1) \sim(7.4)$, it follows that all the hypotheses of Theorem 3.1 are satisfied and this completes the proof of the existence part of Theorem 7.1. For the uniqueness and continuous dependence part, we have only to observe that, in our case,

$$
\|S(\cdot)\|_{L^{1}} \leq \frac{1-e^{-\omega}}{\omega}
$$

and thus, we are in the hypotheses of Theorem 5.1, wherefrom the conclusion.

\section{REFERENCES}

1. Anikonov, Yu. E.; Lorenzi, A. - Explicit representation for the solution to a parabolic differential identification problem in a Banach space, J. Inverse Ill-Posed Probl., 15 (2007), 669-681.

2. Burlic $\breve{A}$, M.; RoşU, D. - A class of nonlinear delay evolution equations with nonlocal initial conditions, Proc. Amer. Math. Soc., in print.

3. Di Blasio, G.; Lorenzi, A. - Identification problems for integro-differential delay equations, Differential Integral Equations, 16 (2003), 1385-1408. 
4. Di Blasio, G.; LoRenzi, A. - Recovering memory kernels in retarded functional differential equations, Dyn. Contin. Discrete Impuls. Syst. Ser. A: Math. Anal., 12 (2005), 837-856.

5. Di Blasio, G.; Lorenzi, A. - Identification problems for parabolic delay differential equations with measurement on the boundary, J. Inverse Ill-Posed Probl., 15 (2007), 709-734.

6. Lorenzi, A.; VRABie, I.I. - An identification problem for a linear evolution equation in a Banach space and applications, Discrete Contin. Dyn. Syst. Ser. S, 4 (2011), 671691.

7. Lorenzi, A.; VRABIE, I.I. - Identification for a semilinear evolution equation in a Banach space, Inverse Problems, 26 (2010), 085009, 16 pp.

8. Lorenzi, A.; VRABie I.I. - An identification problem for a nonlinear evolution equation in a Banach space, Appl. Anal., 91 (2012), 1583-1604.

9. Lorenzi, A.; VRABIE, I.I. - An identification problem for a Semilinear Evolution Delay Equation, submitted for publication.

10. Mitidieri, E.; VRABIE, I.I. - Existence for nonlinear functional differential equations, Hiroshima Math. J., 17 (1987), 627-649.

11. PAZY, A. - Semigroups of Linear Operators and Applications to Partial Differential Equations, Applied Mathematical sciences, 44, Springer Verlag, New York, 1983.

12. VRABIE, I.I. - Compactness methods for an abstract nonlinear Volterra integrodifferential equation, Nonlinear Anal., 5 (1981), 355-371.

13. VRABIE, I.I. - Compactness Methods for Nonlinear Evolutions, Pitman Monographs and Surveys in Pure and Applied Mathematics, 75, Second Edition, Longman John Wiley\&Sons Inc., New York, 1995.

14. VRABIE, I.I. $-C_{0}$-Semigroups and Applications, North-Holland Mathematics Studies, 191, North-Holland Publishing Co., Amsterdam, 2003.

Received: 2.XII.2012

Accepted: 23.I.2013
Dipartimento di Matematica "F. Enriques", Università degli Studi di Milano, Via Saldini 50 20133, Milano,

ITALY

alfredo.lorenzi@unimi.it

Faculty of Mathematics, "Al. I. Cuza"University, Iaşi 700506, ROMANIA

and

Octav Mayer Institute of Mathematics (Romanian Academy),

Iaşi 700505,

ROMANIA

ivrabie@uaic.ro 